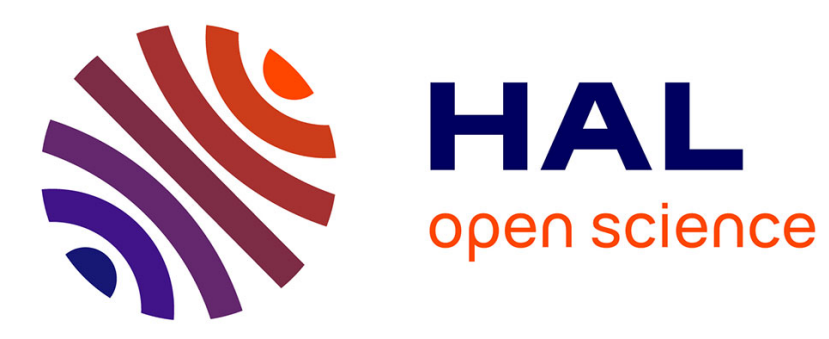

\title{
Refined vorticity statistics of decaying rotating three-dimensional turbulence
}

Laurens J. A. van Bokhoven, Claude Cambon, Lukas Liechtenstein, Fabien Godeferd, H. J. H. Clercx

\section{- To cite this version:}

Laurens J. A. van Bokhoven, Claude Cambon, Lukas Liechtenstein, Fabien Godeferd, H. J. H. Clercx. Refined vorticity statistics of decaying rotating three-dimensional turbulence. Journal of Turbulence, 2008, 9 (6), pp.1-24. 10.1080/14685240701877271 . hal-00517000

\section{HAL Id: hal-00517000 https://hal.science/hal-00517000}

Submitted on 13 Sep 2010

HAL is a multi-disciplinary open access archive for the deposit and dissemination of scientific research documents, whether they are published or not. The documents may come from teaching and research institutions in France or abroad, or from public or private research centers.
L'archive ouverte pluridisciplinaire HAL, est destinée au dépôt et à la diffusion de documents scientifiques de niveau recherche, publiés ou non, émanant des établissements d'enseignement et de recherche français ou étrangers, des laboratoires publics ou privés. 


\title{
Refined vorticity statistics of
}

\section{decaying rotating three-dimensional turbulence}

\author{
L.J.A. van Bokhoven ${ }^{\dagger}$, C. Cambon ${ }^{\ddagger}$, L. Liechtenstein ${ }^{\ddagger}$, F.S. Godeferd ${ }^{\ddagger}$, H.J.H. Clercx ${ }^{\dagger}$
}

(18 October, 2007)

The influence of background rotation on all nontrivial third-order vorticity correlations is studied for an unbounded incompressible homogeneous turbulent flow, using pseudo-spectral direct numerical simulation. The behaviour of third-order vorticity correlations is found to be consistent with exact theoretical predictions presented herein for axisymmetric turbulence without mirror symmetry. Particular attention is given to the vertical vorticity skewness $S_{\omega_{3}}$. Its dependence on the viscosity, the initial value of the velocity gradient skewness, and the background rotation rate has been thoroughly investigated. The initial growth rate of $S_{\omega_{3}}$ provides evidence for a power-law behaviour proportional to $t^{0.75 \pm 0.1}$ for all considered cases, in agreement with recent experimental results by Morize et al. [Phys. Fluids 17, 095105 (2005)]. It is also found that higher background rotation rates - implying more linearity - result in lower final values of $S_{\omega_{3}}$, while lower viscosities and lower initial (absolute) values of the velocity derivative skewness both yield higher final values of $S_{\omega_{3}}$.

Keywords: Decaying homogeneous rotating turbulence; Third-order statistics; Direct Numerical Simulation; Vorticity skewness; Velocity derivative skewness;

$\dagger$ J.M. Burgers Centre and Fluid Dynamics Laboratory, Department of Applied Physics, Eindhoven University of Technology, P.O. Box 513, 5600 MB Eindhoven, The Netherlands. E-mail: 1.j.a.v.bokhoven@tue.nl $\ddagger$ Laboratoire de Mécanique des Fluides et d'Acoustique, École Centrale de Lyon, 69134 Écully Cedex, France. 


\section{Introduction}

Rotating turbulence plays an important role in fields as diverse as geophysics, astrophysics and engineering (e.g. turbomachinery and reciprocating engines with swirl and tumble). In these applications, effects of rotation are often combined with those of mean shear, mean strain and solid boundaries. The problem of homogeneous rotating turbulent flow without physical boundaries must therefore be considered as a canonical flow, even though it stands rather far from applications. A better knowledge of this particular flow is a prerequisite for understanding more complex flows involving rotation.

Here, attention is restricted to the simplest case of rotating homogeneous turbulence, namely unbounded divergence-free flow with zero mean-flow in the co-rotating frame of reference. In this case it is most convenient to adopt a Cartesian coordinate system $\left(x_{1}, x_{2}, x_{3}\right)$ rotating at constant angular velocity $\Omega$. We choose $\Omega=\Omega_{3}>0$ without loss of generality. In this non-Galilean coordinate frame, rotation introduces the well-known centrifugal and Coriolis forces. Since the centrifugal force can be incorporated in the pressure term, only the Coriolis force appears explicitly in the Navier-Stokes equations, viz.

$$
\mathrm{D}_{t} u_{i} \equiv \partial_{t} u_{i}+u_{j} \partial_{j} u_{i}=-\partial_{i} p-2 \Omega \varepsilon_{i 3 k} u_{k}+\nu \partial_{j}^{2} u_{i}
$$

where $\mathrm{D}_{t}$ represents the total derivative, $\partial_{t}$ the partial derivative with respect to time $t$, $\partial_{j}$ the partial derivative with respect to spatial coordinate $x_{j}, u_{i}$ the fluctuating velocity in the co-rotating reference frame, $p$ the corrected pressure divided by a mean reference density, $\varepsilon$ the antisymmetric Levi-Civita tensor, and $\nu$ the kinematic viscosity. Note that a summation is implied over any repeated Roman index unless explicitly stated otherwise. The incompressibility of the flow is expressed by

$$
\partial_{i} u_{i}=0
$$


The modification of turbulence dynamics by rotation is due to the presence of inertial waves in rotating flows [1]. These wave motions arise from the linearised Navier-Stokes equations. In contrast with most turbulent shear flows, however, there is no 'production' of turbulent kinetic energy since the Coriolis force produces no energy. Instead, energy is redistributed by nonlinear mechanisms such as resonant wave interactions. Accordingly, single-point modeling is almost irrelevant to describe the dynamics of rotating turbulence whereas the spectral, or two-point, approach is well adapted (see e.g. $[2,3]$ ).

When a Coriolis force is suddenly imposed on initially isotropic turbulence, the following three main effects are shown. First, the energy cascade is partly inhibited which is linked to a strongly reduced dissipation rate (such an effect can be mimicked by a modification of the dissipation equation [3]). Second, because the dispersion relations for inertial waves are anisotropic, the initial isotropy is broken. This breaking of isotropy is reflected by an incomplete transition from three-dimensional (3D) to two-dimensional (2D) structure. Third, an asymmetry appears between cyclonic and anticyclonic fluctuating vertical (along the rotation axis) vorticity.

The effects mentioned above are intimately connected and result from both linear and nonlinear effects which interplay in a subtle way to drive the dynamics of rotating turbulence. In this paper, we focus on the third effect using rational analysis of relevant exact dynamical equations as well as Direct Numerical Simulation (DNS). As firstly pointed out by Bartello et al. [4], the dominance of cyclonic vorticity can be quantified by the vertical vorticity skewness $S_{\omega_{3}} \equiv\left\langle\omega_{3}^{3}\right\rangle /\left\langle\omega_{3}^{2}\right\rangle^{3 / 2}$, where $\omega_{i} \equiv \varepsilon_{i j k} \partial_{j} u_{k}$ is the vorticity and $\langle\cdot\rangle$ denotes ensemble averaging, since the third-order vorticity correlation $\left\langle\omega_{3}^{3}\right\rangle$ can distinguish by its sign cyclonic prevalence $\left(\omega_{3}>0\right)$ from anticyclonic prevalence $\left(\omega_{3}<0\right)$. Bartello and coworkers found a clear growth of the vertical vorticity skewness using Large Eddy Simulation (LES) with 
hyperviscosity. Although their results are questionable, because vorticity represents small scales and is not correctly captured in a LES, they reflect an actual feature of rotating flows, confirmed experimentally by, among others, Morize et al. [5].

Clearly, the third-order correlation $\left\langle\omega_{3}^{3}\right\rangle$ plays an important role when addressing the asymmetry between cyclonic and anticyclonic vorticity in rotating turbulence. The role of the remaining single-point vorticity triple correlations $\left\langle\omega_{i} \omega_{j} \omega_{k}\right\rangle$ (VTCs hereinafter) is unclear however. Assuming axial symmetry without mirror symmetry, it can be shown very generally that the VTCs involve only two key correlations, viz. $\left\langle\omega_{1}^{2} \omega_{3}+\omega_{2}^{2} \omega_{3}\right\rangle$ and $\left\langle\omega_{3}^{3}\right\rangle$ (see Sect. 2). This theoretical result has been verified with DNS by analysing the time evolution of the complete set of nontrivial third-order vorticity correlations. In addition, we have thoroughly investigated how the vertical vorticity skewness $S_{\omega_{3}}$ is affected by the viscosity, the background rotation rate, and the initial value of the velocity derivative skewness.

Since the dynamics of rotating turbulence is driven by both linear and nonlinear effects, one might wonder what linear theory can say about the VTCs. The so-called Rapid Distortion Theory (RDT) is relevant here, provided that it is developed in Fourier space $[2,6]$ in order to render tractable the non-local relation between pressure and velocity fluctuations, see Appendix A. Accordingly, a general solution in terms of Green's functions can be derived for the VTCs $\left\langle\omega_{i} \omega_{j} \omega_{k}\right\rangle$ at any time $t$, see (A11). This general solution however, is only useful if a full description of all triads of the initial spectral vorticity distribution is at hand. The multifold behaviour of the various triple correlations in the anisotropic case suggests to revisit elaborated Eddy-Damped Quasi-Normal Markovian (EDQNM) theories for recovering such information. For example, basic isotropic EDQNM can be used for initialising the vorticity correlations in the general linear solution for the VTCs (A11). In this sense, anisotropic multipoint statistical theory remains a relevant alternative to DNS, providing access to much higher 
Reynolds numbers and elapsed times - with in counterpart of course less flexibility and the need for statistical assumptions.

This paper is organised as follows. A theoretical analysis of the relevant exact dynamical equations is presented in Sect. 2. A brief description of the numerical algorithm and procedures is given in Sect. 3. In Sect. 4 we present the time evolution of relevant statistical quantities, such as the vorticity intensity production rate (defined in Sect. 2) and the vorticity skewness $S_{\omega_{3}}$. In particular, it is shown that our numerical results are consistent with the theoretical prediction (7). We conclude in Sect. 5 with a summary of our findings and related discussion.

\section{Theory}

The relevant symmetry for rotating turbulence is axial symmetry without mirror symmetry with consequences for any statistical approach, from multi-point [2] to single-point. In this particular case, any tensor related to single-point statistical moments can be expressed in terms of products of the Kronecker symbol $\delta_{i j}$, the axial vector $\mathbf{n}$, and the third-order tensor $\varepsilon_{i j n}$. (For isotropic turbulence, only products of Kronecker symbols are required.) Every tensorial term in the development is weighted by a 'scalar' which depends on the fully contracted scalar product between the relevant vectors. So, the most general development of a single-point third-order tensor $\mathrm{T}$ in the case of axial symmetry without mirror symmetry would be

$$
T_{i j k}=A \delta_{i j} n_{k}+B \delta_{k i} n_{j}+C \delta_{j k} n_{i}+D n_{i} n_{j} n_{k}+F \varepsilon_{i j k}
$$

with the 'scalars' $A, B, C, D$, and $F$ depending only on time, i.e. $A(t)$, etc.

If $\mathrm{T}$ is fully symmetric in terms of its three indices, like e.g. $\left\langle\omega_{i} \omega_{j} \omega_{k}\right\rangle$, then $A=B=C$ 
and $F=0$, so that (3) simplifies to

$$
T_{i j k}=A\left(\delta_{i j} n_{k}+\delta_{k i} n_{j}+\delta_{j k} n_{i}\right)+D n_{i} n_{j} n_{k}
$$

The remaining 'scalars' $A$ and $D$ may be identified by considering particular contracted forms of $T$, for instance

$$
\begin{aligned}
& T_{i i k} n_{k}=5 A+D, \\
& T_{i j k} n_{i} n_{j} n_{k}=3 A+D .
\end{aligned}
$$

From these last two equations it is readily derived that

$$
\begin{aligned}
& A=\left(T_{i i k} n_{k}-T_{i j k} n_{i} n_{j} n_{k}\right) / 2, \\
& D=\left(5 T_{i j k} n_{i} n_{j} n_{k}-3 T_{i i k} n_{k}\right) / 2 .
\end{aligned}
$$

Replacing $T_{i j k}$ by the single-point VTCs $\left\langle\omega_{i} \omega_{j} \omega_{k}\right\rangle$ and setting $n_{i}=\delta_{i 3}$ without loss of generality (index 3 refers to the axial direction) leads to

$$
\left\langle\omega_{i} \omega_{j} \omega_{k}\right\rangle=\left\langle\omega_{3}^{3}\right\rangle n_{i} n_{j} n_{k}+\frac{1}{2}\left\langle\omega_{1}^{2} \omega_{3}+\omega_{2}^{2} \omega_{3}\right\rangle\left(\delta_{i j} n_{k}+\delta_{k i} n_{j}+\delta_{j k} n_{i}-3 n_{i} n_{j} n_{k}\right) .
$$

In other words, in case of axisymmetry without mirror symmetry the VTCs involve only two key correlations, viz. $\left\langle\omega_{3}^{3}\right\rangle$ and $\left\langle\omega_{1}^{2} \omega_{3}+\omega_{2}^{2} \omega_{3}\right\rangle$.

Gence and Frick [7] have shown that for very short times all odd statistical moments of the turbulent vorticity field (except for the first one) are instantaneously sensitive to the influence of mean rotation, while all even moments of the fluctuating vorticity do not change at the first order in time. (For isotropic turbulence, all odd order tensors are zero while a priori non-zero for even order tensors.) The analysis by Gence and Frick concerns the hypothetical case of a homogeneous and isotropic turbulence that is suddenly subjected to solid body rotation. In 
particular, they derive that

$$
\begin{aligned}
\left.\frac{\mathrm{d}}{\mathrm{d} t}\left\langle\omega_{1}^{2} \omega_{3}\right\rangle\right|_{0^{+}}=\left.\frac{\mathrm{d}}{\mathrm{d} t}\left\langle\omega_{2}^{2} \omega_{3}\right\rangle\right|_{0^{+}} & =\frac{4}{15} e(0) \Omega \\
\left.\frac{\mathrm{d}}{\mathrm{d} t}\left\langle\omega_{3}^{3}\right\rangle\right|_{0^{+}} & =\frac{4}{5} e(0) \Omega
\end{aligned}
$$

with the background rotation abruptly initialised at time $t=0$. For details concerning the derivation of (8), the reader is referred to Ref. 7. [It is noted that (8) differs from the original equation (5) in Ref. 7 by a factor of two in the right-hand side because of the unconventional vorticity definition used therein.] Equation (8) states that for times $t=0^{+}$the time derivative of the nontrivial VTCs is proportional to both the rotation rate and the vorticity intensity production rate $e \equiv\left\langle\omega_{i} \omega_{j} s_{i j}\right\rangle$ at $t=0$, with $s_{i j} \equiv \frac{1}{2}\left(\partial_{j} u_{i}+\partial_{i} u_{j}\right)$ the (symmetric) strain rate tensor. In fact, $e$ represents the vortex stretching term in the governing equation of the vorticity intensity $\left\langle\omega^{2}\right\rangle$, so that $e>0$.

It immediately follows from (8) that the nontrivial VTCs will grow and adopt positive values if the rotation rate is positive $(\Omega>0)$. In particular, the probability distribution function of $\omega_{3}$ will become positively skewed, implying that large positive fluctuations in $\omega_{3}$ are more likely to occur than large negative fluctuations in $\omega_{3}$. The result (8) also reflects the fact that the complex nonlocal effect of pressure fluctuation, apparent from the linearised Poisson equation [see (A2) in Appendix A], is not involved at the first order of a Taylor expansion in time, so that a simplified solution can be found in physical space.

The above result can be explained from a more general viewpoint, starting from the basic equation for the fluctuating vorticity,

$$
\mathrm{D}_{t} \omega_{i}=\omega_{j} s_{i j}+2 \Omega \partial_{3} u_{i}+\nu \partial_{j}^{2} \omega_{i}
$$

obtained by taking the curl of the Navier-Stokes equations (1). The first term on the right- 
hand side of (9) represents vorticity production due to nonlinear vortex stretching, whereas the second term represents its linear counterpart, which is specifically induced by solid body rotation. From the vorticity equation (9), one may easily obtain the governing equations for the single-point VTCs $\left\langle\omega_{i} \omega_{j} \omega_{k}\right\rangle$. Under the sole assumption of homogeneity, i.e. $\left\langle\mathrm{D}_{t} \boldsymbol{\omega}\right\rangle \equiv$ $\mathrm{d}\langle\boldsymbol{\omega}\rangle / \mathrm{dt}$, one derives that

$$
\begin{aligned}
\frac{\mathrm{d}}{\mathrm{d} t}\left\langle\omega_{i} \omega_{j} \omega_{k}\right\rangle=\left\langle\omega_{i} \omega_{j} \omega_{q} s_{k q}\right\rangle & +\left\langle\omega_{j} \omega_{k} \omega_{q} s_{i q}\right\rangle \\
+2 \Omega\left(\left\langle\omega_{i} \omega_{j} \partial_{3} u_{k}\right\rangle\right. & \left.+\left\langle\omega_{k} \omega_{i} \omega_{q} \omega_{k} \partial_{3} u_{i}\right\rangle+\left\langle\omega_{k} \omega_{i} \partial_{3} u_{j}\right\rangle\right) \\
& +\nu\left(\left\langle\omega_{i} \omega_{j} \partial_{q}^{2} \omega_{k}\right\rangle+\left\langle\omega_{j} \omega_{k} \partial_{q}^{2} \omega_{i}\right\rangle+\left\langle\omega_{k} \omega_{i} \partial_{q}^{2} \omega_{j}\right\rangle\right) .
\end{aligned}
$$

This equation can be considerably simplified under the additional assumption of axial symmetry without mirror symmetry. The key correlations involved [see Eq. (7)] are governed by

$$
\begin{aligned}
\frac{\mathrm{d}}{\mathrm{d} t}\left\langle\omega_{\alpha}^{2} \omega_{3}\right\rangle=\left\langle\omega_{\alpha}^{2} \omega_{q} s_{3 q}\right\rangle+2\left\langle\omega_{3} \omega_{\alpha} \omega_{q} s_{\alpha q}\right\rangle+ & \\
2 \Omega\left(\left\langle\omega_{\alpha}^{2} s_{33}\right\rangle+2\left\langle\omega_{3} \omega_{\alpha} s_{\alpha 3}\right\rangle\right) & \\
& +\nu\left(\left\langle\omega_{\alpha}^{2} \partial_{q}^{2} \omega_{3}\right\rangle+2\left\langle\omega_{3} \omega_{\alpha} \partial_{q}^{2} \omega_{\alpha}\right\rangle\right),
\end{aligned}
$$

and

$$
\frac{\mathrm{d}}{\mathrm{d} t}\left\langle\omega_{3}^{3}\right\rangle=3\left\langle\omega_{3}^{2} \mathrm{D}_{t} \omega_{3}\right\rangle=3\left\langle\omega_{3}^{2} \omega_{q} s_{3 q}\right\rangle+6 \Omega\left\langle\omega_{3}^{2} s_{33}\right\rangle+3 \nu\left\langle\omega_{3}^{2} \partial_{q}^{2} \omega_{3}\right\rangle
$$

respectively, where the index summation convention does not apply to the Greek index $\alpha=1,2$. In deriving (11) the relationship $\partial_{j} u_{i}=s_{i j}+\frac{1}{2} \varepsilon_{i k j} \omega_{k}$ has been used, and also the fact that $\left\langle\omega_{1} \omega_{2} \omega_{3}\right\rangle=0$ in case of axial symmetry without mirror symmetry [see Eq. (7)]. The terms $2 \Omega(\cdot)$ on the right-hand side of equations (11) reflect the specific rotation-induced produc- 
tion. They appear directly proportional to componential vortex stretching terms, viz. $\left\langle\omega_{\alpha}^{2} s_{33}\right\rangle$ and $\left\langle\omega_{3} \omega_{\alpha} s_{\alpha 3}\right\rangle$ in (11a), and $\left\langle\omega_{3}^{2} s_{33}\right\rangle$ in (11b), respectively. Throughout this paper the terms $\left\langle\omega_{i} \omega_{j} s_{k l}\right\rangle$ are referred to as 'componential vortex stretching terms'. They should not be confused with the vortex stretching terms $\omega_{j} s_{i j}$ in (9).

Note that the result of Gence and Frick (8) at the first order of time immediately follows from (11) under the assumption of statistically isotropic initial data. After all, in case of isotropy the componential vortex stretching terms (e.g. $\left.\left\langle\omega_{3}^{2} s_{33}\right\rangle\right)$ may be derived from the vorticity intensity production rate $e=\left\langle\omega_{i} \omega_{j} s_{i j}\right\rangle$, via exact coefficients:

$$
\left\langle\omega_{i} \omega_{j} s_{k l}\right\rangle=\frac{e}{10}\left(\delta_{k i} \delta_{l j}+\delta_{k j} \delta_{l i}-\frac{2}{3} \delta_{k l} \delta_{i j}\right)
$$

The advantage of (11) above (8) is to display how specific components of vortex stretching relate to specific components of VTCs. For example, it follows from (11b) that the rotationinduced growth of $\left\langle\omega_{3}^{3}\right\rangle$ is fully controlled by the componential vortex stretching term $\left\langle\omega_{3}^{2} s_{33}\right\rangle$. A complete physical explanation for the link between the componential vortex stretching term and the velocity derivative skewness is still lacking, except for the isotropic case. In that case, one has the isotropic relationship

$$
\left\langle\omega_{3}^{2} s_{33}\right\rangle=\frac{2}{15} e=-\frac{2}{15} \sqrt{\frac{98}{135}} W^{3 / 2} S_{\partial_{3} u_{3}}
$$

with $W \equiv \frac{1}{2}\left\langle\omega_{i}^{2}\right\rangle$ the global enstrophy. [Relationship (13) is discussed in detail in Sects 3 and 4.3.] As a result, $\left\langle\omega_{3}^{2} s_{33}\right\rangle$ is positive if the velocity derivative skewness $S_{\partial_{3} u_{3}} \equiv$ $\left\langle\left(\partial_{3} u_{3}\right)^{3}\right\rangle /\left\langle\left(\partial_{3} u_{3}\right)^{2}\right\rangle^{3 / 2}$ is negative - provided the flow is significantly 3D. Roughly speaking, the fact that the velocity derivative skewness is significantly negative in turn characterises a direct cascade, in close connection with a negative third-order structure function at the Taylor microscale. On the other hand, if the flow were closer to a $2 \mathrm{D}$ limit, $s_{33}$ might be 
strongly reduced, and so would the rotation-induced rate of production of $\left\langle\omega_{3}^{3}\right\rangle-$ all other quantities remaining unchanged.

\section{Numerical procedures}

Equations (1) and (2) are solved directly using a pseudo-spectral collocation method expressed in Fourier space following a classical scheme as in e.g. [8-10]. The computational domain uses periodic boundary conditions, and consists of $N^{3}$ points in physical space. Furthermore, the velocity field is completely de-aliased using a 2/3-truncation method in Fourier space. At time $t=0$, the velocity field is initialised with the narrow band energy spectrum $E(k) \propto$ $k^{4} \mathrm{e}^{-2\left(k / k_{i}\right)^{2}}$, with $k$ the wave number. The energy spectrum $E(k)$ is maximum at wave number $k_{i}$, typically one tenth of the maximum wave number of the de-aliased field. The directions of the initial velocity vectors are distributed randomly in space and so do not correlate with each other after initialisation. From this initially random isotropic field an isotropic precalculation is performed up to time $t_{\text {ini }}$ in order to allow for higher order velocity correlations to develop and isotropic energy dynamics to build up. Various Eulerian temporal statistics are calculated during the simulation at predefined time steps.

At $t=t_{\text {ini }}$, the background rotation $\Omega=(0,0, \Omega)$ is suddenly imposed upon the welldeveloped isotropic turbulent velocity field. The related discontinuities at time $t_{\text {ini }}$ in the temporal derivatives of spatially averaged quantities, such as the vorticity skewness, are ignored. In order to check consistency with the theoretical result (7), the minimal set of components describing all VTCs in rotating turbulence is calculated for $t>t_{\text {ini }}$, viz. $\left\langle\omega_{1}^{3}\right\rangle$, $\left\langle\omega_{1}^{2} \omega_{3}\right\rangle,\left\langle\omega_{1} \omega_{3}^{2}\right\rangle,\left\langle\omega_{1} \omega_{2} \omega_{3}\right\rangle,\left\langle\omega_{2}^{2} \omega_{3}\right\rangle$, and $\left\langle\omega_{3}^{3}\right\rangle$. In all our numerical results any ensemble average has been approximated by a spatial average taken over the entire computational domain. The spatial average is also denoted by the angular brackets $\langle\cdot\rangle$. 
We have performed anisotropic computations with different (nondimensional) kinematic viscosities $\nu$ and for various background rotation rates (as expressed by the Coriolis parameter $f \equiv 2 \Omega$ ) in order to quantify how these parameters affect the temporal evolution of the VTCs. An overview of the performed runs and their parameters is presented in Table 1. Let $\lambda \equiv\left(15 u^{\prime 2} /\left\langle\omega_{i}^{2}\right\rangle\right)^{1 / 2}$ represent the Taylor microscale, with $u^{\prime} \equiv\left\langle u_{i}^{2} / 3\right\rangle^{1 / 2}$ the characteristic velocity. We remark that the Taylor-scale based Reynolds number $R e_{\lambda} \equiv u^{\prime} \lambda / \nu$ is not maintained in decaying turbulence, so that $R e_{\lambda}$ depends on $t_{\text {ini }}$.

The time $t_{\text {ini }}$ at which anisotropy is introduced is a crucial parameter. In a conventional DNS (such as ours), any third-order correlation is almost zero initially in connection with the (close to) Gaussian initialisation. During the subsequent (isotropic) precalculation certain triple correlations develop, for instance creating a significant velocity derivative skewness $S_{\partial_{3 u_{3}}}$. The latter can asymptote to a negative value, reflecting a nondimensional constant rate of enstrophy production by nonlinear vortex stretching since the normalised vortex stretching term

$$
e^{*} \equiv \sqrt{\frac{135}{98}} \frac{\left\langle\omega_{i} \omega_{j} s_{i j}\right\rangle}{W^{3 / 2}}=-S_{\partial_{3} u_{3}}
$$

in case of isotropy [11]. Other triple correlations such as the cubic vertical vorticity $\left\langle\omega_{3}^{3}\right\rangle$ remain zero because of isotropy. At $t=t_{\mathrm{ini}}$, the isotropy is broken by rotation and certain VTCs will develop [see (7) or (8)]. The velocity derivative skewness and the vortex stretching term $e^{*}$, on the other hand, will be rapidly and severely damped. The exact mechanism that causes certain VTCs to grow in time is still unknown. Nevertheless, the work by Gence and Frick [7] strongly suggests that the growth of certain VTCs is caused by linear mechanisms. If a purely linear mechanism is assumed, then the maximum growth of the cubic vertical vorticity $\left\langle\omega_{3}^{3}\right\rangle$ will depend on the level of non-Gaussianity reached at the end of the precalculation, 
with two extremes: on the one hand, a very short precalculation implying small departure from Gaussianity at $t=t_{\text {ini }}$ so that the initial peak of $\left\langle\omega_{3}^{3}\right\rangle$ will be small too, and on the other hand, a long precalculation implying maximum level of non-Gaussianity at $t=t_{\text {ini }}$ so that the initial peak of $\left\langle\omega_{3}^{3}\right\rangle$ will be maximum.

Since the velocity derivative skewness clearly illustrates the departure from Gaussianity of the velocity fluctuations, reaching values of approximately -0.5 in isotropic turbulence [12], it seems an obvious variable from which to derive time $t_{\text {ini }}$. However, only in case of isotropy — so during the precalculation — the velocity derivative skewness can be directly related to vortex stretching [see (14)]. For times $t \geq t_{\text {ini }}$, the imposed background rotation introduces anisotropy. The isotropic link between the velocity derivative skewness and vortex stretching then no longer applies, and physical interpretation of the velocity derivative skewness is complicated. Therefore, we prefer to derive $t_{\text {ini }}$ from the time evolution of $e^{*}$, which has a clear physical meaning regardless of the turbulence state. Accordingly, it seems obvious to choose time $t_{\text {ini }}$ such that $e^{*}$ is maximum. However, during a precalculation of decaying turbulence a significant part of the turbulent kinetic energy is lost due to dissipation. In order to have a sufficiently turbulent flow field at the end of the isotropic precalculation, time $t_{\text {ini }}$ is chosen such that $e^{*}$, and so also the velocity derivative skewness, has almost reached its final value. How the choice of $t_{\mathrm{ini}}$ affects the relevant statistics is discussed in Sect. 4.

\section{Numerical results}

In what follows we present the time evolution of various Eulerian quantities during the anisotropic computation unless stated otherwise. Wherever convenient we use the scaled, shifted time $\tau \equiv\left(t-t_{\text {ini }}\right) f /(2 \pi)$, with $f \equiv 2 \Omega$ the Coriolis parameter, to simplify the comparison between runs for which anisotropy is introduced at distinct times $t_{\text {ini }}$, and also to 
simplify the comparison with recent experimental results $[5,13]$.

We first investigate how viscosity affects the time evolution of the kinetic energy and relevant nondimensional numbers in presence of background rotation. Beforehand, it is remarked that the energy decay in absence of rotation usually is well described by a power-law $t^{-n}$. Theoretical predictions range from $n=6 / 5$ for unbounded turbulence [14] to $n=2$ for domain-bounded turbulence [15]. Exponents in the range 1-1.4 are encountered in the literature for wind-tunnel experiments. Figure 1 shows a natural plot of the (monotonic) decay of the kinetic energy $E \equiv \frac{1}{2}\left\langle u_{i}^{2}\right\rangle$ for various viscosities $\nu$. Rotation is known to inhibit the energy transfer to the viscous scales, and the energy decay rate is seen to be reduced accordingly. In order to gain insight into the power-law behaviour of the kinetic energy a plot on logarithmic scales is needed. The runs depicted in Fig. 1 however, are inadequate for investigating the asymptotic time limit $t \gg t_{\text {ini }}$ as they cover too short a time period. (The present study is focussed on the initial behaviour of third-order statistics, so that most computations only concern the initial period of decay.) Instead, consider cases C1 and C2. These computations have been specially prolonged to study the long-time asymptotic behaviour of the kinetic energy. Figure 2 shows a doubly logarithmic plot of the energy decay in the isotropic case (labeled Cref) and in the rotating cases $\mathrm{C} 1$ and $\mathrm{C} 2$, respectively. In absence of rotation, the energy decay is proportional to the power-law $t^{-10 / 7}$, consistent with the above-mentioned predictions. In presence of rotation, the energy decay rate appears to incline towards the power-law $t^{-5 / 7}$ in the asymptotic limit $t \gg t_{\text {ini }}$. The energy decay rate in the rotating case is thus reduced by a factor two relative to its value in the non-rotating case. This result is in perfect agreement with the numerical and theoretical analysis by Squires et al. [16]. Moreover, the exponent $n=5 / 7$ for the asymptotic energy decay in presence of rotation could indicate a $k^{-2}$ energy spectrum for large wave numbers $[17,18]$. 
Next, consider the time dependence of the nondimensional numbers $\operatorname{Re}_{\lambda}$ and $R o_{\lambda} \equiv$ $u^{\prime} /(2 \Omega \lambda)$, see Figs $3(\mathrm{a})$ and (b), respectively. In contrast with the decrease of $\operatorname{Re}_{\lambda}$ during the isotropic precalculation (not shown), Fig. 3(a) shows that sufficiently strong background rotation (as in cases $\mathrm{A}, \mathrm{C} 3$ and $\mathrm{D}$ ) results in a growth of $R e_{\lambda}$. This implies that the Taylor microscale $\lambda$ grows faster than the kinetic energy decays. The Taylor-based Rossby number decays monotonically [Fig. 3(b)], which is characteristic for decaying rotating turbulence $[3,19,20]$. Furthermore, $R o_{\lambda}<1$ for all times and in all cases which means that background rotation is dominant over nonlinear effects for all times and in all cases.

Similar time evolutions of $E, R e_{\lambda}$ and $R o_{\lambda}$ have been obtained for the remaining runs (not shown) with one exception: for the lowest background rotation rate considered here (case C1) the Taylor-based Rossby number $R_{\lambda}\left(t_{\text {ini }}\right)>1$, which illustrates that background rotation is not yet dominant over nonlinear effects (so-called weakly rotating turbulence). In this particular case, $R e_{\lambda}$ initially continues to fall off with time.

We now discuss the effect of rotation on the energy spectrum. Figure 4 shows the energy spectrum at the start $\left(t=t_{\text {ini }}\right)$ as well as at the end $\left(t=t_{f}\right)$ of the anisotropic computation. From left to right, the panels correspond to cases A, C3, and D, respectively. The energy spectrum at time $t_{f}$ for the corresponding isotropic (reference) computations is also plotted. Comparison of the energy spectra at time $t_{f}$ for every reference and rotating case reveals that $E(k)$ is almost conserved in presence of rotation for $k<k_{i}$ while steeper for wave numbers $k>k_{i}$. Rotation inhibits the direct energy cascade in the inertial range, i.e. energy transfer towards the larger wave numbers is reduced, and so energy is almost conserved at the smaller wave numbers. In addition, note that none of the energy spectra in Fig. 4 display clear powerlaw behaviour in the inertial range, neither in absence nor in presence of rotation. Taking into consideration that all cases concern decaying turbulence, hence quite low Reynolds number, 
this result is not very surprising.

It follows that our low Reynolds number results show a clear steepening of the slopes of the spectra, induced by rotation, but that they give no definitive quantitative information to compare to classical power laws predicted or obtained at high Reynolds number. Nevertheless, it is useful to briefly discuss such power laws as follows. Yang and Domaradzki [24] found

a transition from $E(k) \sim k^{-2}$ (in agreement with the scaling $E(k) \sim \sqrt{\epsilon \Omega} k^{-2}$ [18], with $\epsilon$ the energy dissipation rate) at moderate rotation rates, to $E(k) \sim k^{-3}$ at the largest elapsed times and highest rotation rates. The latter prediction is not consistent with purely 2D rotating turbulence in which $E(k) \sim \Omega^{2} k^{-3}$, but agrees with theoretical and numerical results in wave-turbulence $[25,26]$. Slopes between -2 and -3 have also been found by Morize et al. [17], and the experimental investigation by Van Bokhoven [27] supports a slope of -3 . Both measurements, however, only give access to a spectrum $E\left(k_{h}\right)$, with $k_{h}$ the horizontal wave number, which is closer (without being the same) to the conventional one-dimensional spectrum based on $u_{1}$ or $u_{2}$ than to the spherically averaged energy spectrum $E(k)$. If the turbulence is strongly anisotropic, the slope in the inertial range can differ for these two types of spectra.

\subsection{Vortex stretching term and vorticity skewness}

The time evolution of the (normalised) vortex stretching term $e^{*}[$ see (14)] and the vorticity skewness $S_{\omega_{3}}$ in cases A, C3 and D (so different viscosities) is depicted in Fig. 5. Clearly, the vortex stretching term develops during the isotropic precalculation $\left(t \leq t_{\text {ini }}\right)$ and collapses when the anisotropic body force is applied $\left(t>t_{\text {ini }}\right)$. Furthermore, it is seen that higher viscosities (i.e. lower spatial resolution) require longer precalculations to attain the same value of $e^{*}$. In cases $\mathrm{A}$ and $\mathrm{C} 3$, the initial increase of $S_{\omega_{3}}$ [Fig. 5(b)] is found to be proportional 
to the power-law $\tau^{0.75 \pm 0.1}$. This power-law behaviour is less apparent in case D, most likely related to the short precalculation as argued further below. For later times $[\tau \gtrsim 1], S_{\omega_{3}}$ wiggles about a positive value, reflecting the dominance of cyclonic vorticity. Figure 5(b) reveals that a lower viscosity results in a higher final value of $S_{\omega_{3}}$. This implies (see Table 1) that if both $R e_{\lambda}\left(t_{\mathrm{ini}}\right)$ and $R o_{\lambda}\left(t_{\mathrm{ini}}\right)$ are higher - so a lower degree of linearity - then the final value of $S_{\omega_{3}}$ will be higher. Although it seems likely that a lower viscosity $\nu$ results in a more developed vorticity skewness, the different final values of $S_{\omega_{3}}$ may as well be ascribed to slight differences in $e^{*}\left(t_{\text {ini }}\right)$, see below.

Consider the effects of the duration of the isotropic precalculation on the time evolution of $S_{\omega_{3}}$. It immediately follows from Fig. 6(a) that shorter precalculations yield smaller $e^{*}\left(t_{\text {ini }}\right)$. The vorticity skewness $S_{\omega_{3}}$ [Fig. 6(b)] shows evidence of power-law behaviour proportional to $\tau^{0.75 \pm 0.1}$ for $\tau \ll 1$, and wiggles about a positive value for $\tau \gtrsim 1$. Moreover, careful analysis of Fig. 6(b) suggests that the initial $(\tau \ll 1)$ power-law behaviour is affected by the duration of the precalculation: both very short and very long precalculations result in a smaller powerlaw exponent. It thus seems likely that the less apparent power-law behaviour of $S_{\omega_{3}}$ in case D [Fig. 5(b)] is related to the short duration of the precalculation. Returning to Fig. 6(b), the amplitude of the initial peak of $S_{\omega_{3}}$ is seen to depend on the initial Reynolds and Rossby number in such a way that if both $R e_{\lambda}\left(t_{\text {ini }}\right)$ and $R o_{\lambda}\left(t_{\text {ini }}\right)$ are decreased by increasing $t_{\text {ini }}$, this results in a larger peak amplitude. Furthermore, the final value of $S_{\omega_{3}}$ appears to depend on $t_{\text {ini }}$ in the following manner: shorter precalculations, implying higher $\operatorname{Re}_{\lambda}\left(t_{\mathrm{ini}}\right)$ and $R o_{\lambda}\left(t_{\mathrm{ini}}\right)$, result in higher final values of $S_{\omega_{3}}$. Thus, as already noted, the behaviour displayed in Fig. 5(b) may partly be ascribed to slight differences in $e^{*}$ at time $t_{\text {ini }}$.

Finally, Fig. 7 shows the time evolution of $e^{*}$ and $S_{\omega_{3}}$ for various background rotation rates, viz. $f \equiv 2 \Omega=0.5 \pi, 2.5 \pi, 5.0 \pi$ and $10.0 \pi$ (cases $\mathrm{C} 1-\mathrm{C} 4$ ). Except for case C1, where no 
clear initial peak is observed, it follows that the amplitude of the initial peak of $S_{\omega_{3}}$ depends on the initial Rossby number, such that a smaller $R o_{\lambda}\left(t_{\mathrm{ini}}\right)$ yields a larger peak amplitude. Furthermore, Fig. 7 shows that a lower background rotation rate - implying higher $R o_{\lambda}\left(t_{\mathrm{ini}}\right)$ — results in a larger final value of $S_{\omega_{3}}$. This result expresses the fact that the asymmetry between cyclonic and anticyclonic structures is more pronounced at low rotation rates than at high rotation rates. Furthermore, it is useful to remark that similar results have been obtained with lower resolution $(N=144)$ calculations.

\subsection{Single-point third-order vorticity correlations and pdfs of vorticity}

Figure 8 shows the time evolution of all nontrivial VTCs for various background rotation rates. The following three observations are made: i) the normalised quantities $\left\langle\omega_{1}^{3}\right\rangle /\left\langle\omega_{3}^{2}\right\rangle^{3 / 2}$, $\left\langle\omega_{1} \omega_{3}^{2}\right\rangle /\left\langle\omega_{3}^{2}\right\rangle^{3 / 2}$ and $\left\langle\omega_{1} \omega_{2} \omega_{3}\right\rangle /\left\langle\omega_{3}^{2}\right\rangle^{3 / 2}$ are much smaller than unity and fluctuate around zero; ii) $\left\langle\omega_{1}^{2} \omega_{3}\right\rangle,\left\langle\omega_{2}^{2} \omega_{3}\right\rangle$ and $\left\langle\omega_{3}^{3}\right\rangle$ are clearly nonzero; and iii) the ratio $\left\langle\omega_{1}^{2} \omega_{3}\right\rangle /\left\langle\omega_{2}^{2} \omega_{3}\right\rangle$ (not shown) is found to fluctuate around unity. These results are all consistent with relationship (7).

Additional information concerning the vorticity field may be derived from the probability distribution functions (pdfs) of the vorticity components, denoted by $p\left[\omega_{i}(t)\right]$. The pdf $p\left(\omega_{3}\right)$ is of particular interest in consideration of the rotation-induced asymmetry between cyclonic and anticyclonic structures. At a given instance of time, the pdfs $p\left(\omega_{i}\right)$ may be obtained by binning the values of $\omega_{i}(\mathbf{x})$ for all space points $\mathbf{x}$ to some relevant predefined range of vorticity values - here, the range $\left[\min \left(\omega_{i}\right), \max \left(\omega_{i}\right)\right]$ is divided into 50 bins. The tails of the pdfs have been slightly smoothed to suppress noise effects. In what follows, the time dependence of the pdfs of vorticity is studied for decaying homogeneous turbulence, both with and without rotation.

To start with, consider the time dependence of $p\left(\omega_{i}\right)$ in the isotropic case Cref [Fig. 9(a)]. 
The thick and thin lines represent $p\left(\omega_{i}\right)$ (consult the legend of the mentioned figure for details) at $t=4$ and $t=8$, respectively. For a given time, the pdfs of different vorticity components collapse which illustrates the isotropy of the turbulence. Furthermore, the purely decaying turbulence behaves in a self-similar manner. After all, consider the normalised pdf $p\left(\omega_{i}\right) \sigma\left(\omega_{i}\right)$ as a function of $\omega_{i} / \sigma\left(\omega_{i}\right)$, with $\sigma\left(\omega_{i}\right)$ the standard deviation of the distribution $p\left(\omega_{i}\right)[$ Fig. $9(\mathrm{~b})]$. The normalised pdfs associated with distinct times collapse for the range $\left|\omega_{i}\right| \leq 5 \sigma\left(\omega_{i}\right)$

The above procedure has been repeated for cases $\mathrm{C} 1$ and $\mathrm{C} 2$ to study the effect of rotation on a decaying (initially isotropic) vorticity distribution. The pdfs have now been computed for times $t=t_{\mathrm{ini}}=4$ (already discussed above) and $t=t_{r}>t_{\mathrm{ini}}$, where $t_{r}$ represents the time at which the Taylor-based Reynolds number reaches a value of approximately 44. (Perhaps needless to remark, but time $t_{r}$ varies with the rotation rate.) By matching the Reynolds number rather then the numerical or scaled time, one simplifies the comparison between cases with different rotation rates, addressed further on. Panels (a) and (b) of Fig. 10 show the normalised pdfs of vorticity for cases $\mathrm{C} 1$ and C2, respectively. [Note that Fig. 9(b) cannot be directly compared to either panel of Fig. 10 because of the difference in Reynolds number.] At time $t_{r}$, the normalised pdfs of the horizontal vorticity components [Fig. 10(a,b)] still collapse despite the presence of rotation. Their shape has slightly changed though compared to time $t_{\text {ini }}$. It is important to note that the horizontal vorticity field has not developed any significant nonzero skewness [see also Fig. 8(a)]. The normalised pdf of the vertical vorticity component (chain-dotted line), on the other hand, reveals an unmistakable positive skewness [see also Fig. 8(f)]. In other words, cyclonic vorticity prevails over anticyclonic vorticity in decaying homogeneous rotating turbulence. Furthermore, comparison of the tails of the vorticity distributions at various well-defined times during the decay strongly suggests 
that the tails are not well-defined in time in case C1 (not shown). This could explain the pronounced wiggly behaviour of the VTCs in case C1 (Fig. 8).

Finally, consider the rotation dependence of the normalised pdfs of vorticity (Fig. 11). The instantaneous Taylor-based Reynolds number is 33.2, and 44.8, 45.5, 45.0, 43.1 in cases Cref, and $\mathrm{C} 1-\mathrm{C} 4$, respectively. The following behaviour is observed as the rotation rate is increased: i) the normalised pdfs of the horizontal vorticity components remain non-skewed, and ii) they tend to converge to a more restricted range of vorticity values; iii) the normalised pdf of the vertical vorticity component becomes less skewed, merely from the evolution of the tail of the pdf in the cyclonic range of vorticity, whereas the tail corresponding to anti-cyclonic vorticity remains unchanged under the effect of rotation.

\subsection{Componential velocity derivative skewnesses}

As discussed before, the velocity derivative skewness develops during the isotropic precalculation and collapses as soon as the isotropic turbulence is subjected to background rotation. In connection with the introduced anisotropy, it is of interest to look at the componential veloc-

ity derivative skewnesses $S_{\partial_{\alpha} u_{\alpha}} \equiv\left\langle\left(\partial_{\alpha} u_{\alpha}\right)^{3}\right\rangle /\left\langle\left(\partial_{\alpha} u_{\alpha}\right)^{2}\right\rangle^{3 / 2}$ (the index summation convention does not apply to the Greek index $\alpha=1,2,3)$. The time evolution of $-S_{\partial_{a} u_{\alpha}}$ is depicted in Fig. 12 for each of the cases given in Table 1. The left column of panels in Fig. 12 corresponds to $-S_{\partial_{1} u_{1}}$ and $-S_{\partial_{2 u_{2}}}$, the right column of panels to $-S_{\partial_{3} u_{3}}$. The minus sign is introduced to enable direct comparison with the vortex stretching term $e^{*}$. The temporal behaviour of $-S_{\partial_{\alpha} u_{\alpha}}(\alpha=1,2$, or 3$)$ is very similar to that of $e^{*}$ for any case [compare Fig. 12 to Figs $5-$ $7(\mathrm{a})]$, except for case $\mathrm{C} 1$. There, $-S_{\partial_{3} u_{3}}$ continues to grow even after the anisotropic body force has been initialised. This behaviour is likely not just a matter of adjustment, but rather a reflection of the nonlinear energy transfers that are abundantly present in the dynamics at 
lower rotation rates.

A closer look at Fig. 12 reveals that the decay curves of $-S_{\partial_{1} u_{1}}$ and $-S_{\partial_{2} u_{2}}$ collapse for times $t_{\text {ini }}^{+}$regardless which case. This initial decay stage thus appears to be independent of the flow history which could suggest some universality of this decay law. However, such a collapse is not found for $-S_{\partial_{3} u_{3}}$, so along the rotation axis. Instead, a gap is observed between the decay curves of $-S_{\partial_{3} u_{3}}$ and those of $-S_{\partial_{1} u_{1}}$ and $-S_{\partial_{2} u_{2}}$. Although this gap might well be related to the structuring of the fluid into columnar structures parallel to the rotation axis, one cannot a priori relate effects observed for a nondimensional ratio such as the skewness to trends of two-dimensionalisation. A partial transition from a 3D to $2 \mathrm{D}$ state would imply a decrease of any $x_{3}$-derivative with respect to its $x_{1}$ counterpart, and so only dimensional quadratic or cubic products of velocity gradients could serve as 2D indicators.

\section{Summary and discussion}

Summarising the main points of this research, it has been derived from exact dynamical equations that the VTCs in an axisymmetric homogeneous turbulence without mirror symmetry involve only two key correlations, see (7). This particular symmetry is relevant to rotating turbulence. The newly derived result has been extensively checked with DNS for decaying homogeneous rotating turbulence without boundaries. Our numerical findings are summarised and discussed in what follows.

First of all, in most of the cases considered here the vertical vorticity skewness $S_{\omega_{3}}$ initially grows at a rate proportional to $\tau^{0.75 \pm 0.1}\left(\sim t^{0.75 \pm 0.1}\right)$. Such power-law behaviour is in good agreement with that observed in recent laboratory experiments $[5,13,21]$, viz. $t^{0.7 \pm 0.1}$ (see also Figs 13 and 14$)$. Moreover, the time evolution of $S_{\omega_{3}}$ in case $\mathrm{C} 1(f \approx 1.6)$ is remarkably similar to the evolution observed by Staplehurst et al. [21] in their experiments on decaying 
rotating homogeneous turbulence (in particular the experiment with $f \approx 1.88$ and bar size $M=32 \mathrm{~mm}$ ), see Fig. 14 .

Nevertheless, our numerical results differ from the experimental results of Morize et al. [5] at two points. First, the amplitude of the peak in the time evolution of $S_{\omega_{3}}$ as well as the (scaled) time at which this peak occurs are significantly smaller in our numerical computations than in the mentioned laboratory experiments [compare Figs 5-7(a) to Fig. 13]. Second, for times $\tau \gtrsim 1$ our results show that the vertical vorticity skewness fluctuates about a positive value — indicating a steady vorticity asymmetry. Morize et al. [13], on the other hand, observe a monotonic decay of $S_{\omega_{3}}$ - implying re-symmetrisation of the vertical vorticity distribution. These differences may be understood as follows. Morize et al. [13] have shown that the peak properties are affected by the vertical confinement of the fluid (linear Ekman pumping effects). Furthermore, they have ascribed the monotonic decay of $S_{\omega_{3}}$ to nonlinear Ekman effects as these enhance the damping of the cyclonic vortices [22]. Since a three-dimensional periodic box is used in our computations, Ekman effects (both linear and nonlinear) obviously play no role and a steady positive vorticity asymmetry can establish for times $\tau \gtrsim 1$, see e.g. Figs $5^{-}$ $7(b)$.

The effects of confinement are perhaps overestimated by Morize et al., especially when they put forward that confinement affects the early stage, i.e. far before the 'inhomogeneous' threshold time. Contrasting with the results of Staplehurst et al. allows to relativise a too strong — and early — inhomogeneous effect. It follows that the absence of confinement in our DNS indeed provides some explanation for the differences between our DNS and the laboratory experiments by Morize and coworkers. One way of testing this conjecture would be to perform a similar study like the one presented here but then for vertically bounded rather than unbounded turbulence. Such is considered beyond the scope of the present research, 
but the issue will be addressed in future studies. Besides Ekman effects, we anticipate that differences in the initial Taylor-based Reynolds number and/or Rossby number also contribute to the above-mentioned differences between DNS and laboratory experiments, as will be explained now.

We have also investigated the dependence of the vertical vorticity skewness on the viscosity, the value of the velocity derivative skewness at time $t_{\text {ini }}$, and the background rotation rate. Each of these parameters affects the initial Taylor-based Rossby number $R o_{\lambda}\left(t_{\mathrm{ini}}\right)$. In addition, the first two parameters also affect the initial Taylor-based Reynolds number $\operatorname{Re}_{\lambda}\left(t_{\text {ini }}\right)$. The obtained results lead to the following general conclusion: lower $\operatorname{Re}_{\lambda}\left(t_{\mathrm{ini}}\right)$ and/or lower $R o_{\lambda}\left(t_{\mathrm{ini}}\right)$ - implying a higher degree of linearity - yield(s) a lower final vorticity skewness. This result confirms that the asymmetry between cyclonic and anticyclonic vorticity is most prominently present in an intermediate range of Rossby numbers, as already discussed by Jacquin et al. [20] for the anisotropic development of integral length scales, and more recently by Bourouiba and Bartello [23] in the context of VTCs. If the Rossby number is too small, nonlinearity is not important enough - even if purely linear dynamics can induce a transient growth of $S_{\omega_{3}}$, that same dynamics results in damping $S_{\omega_{3}}$ at later times. The opposite case of very large Rossby number is not addressed here, but recall that isotropy is conserved and therefore asymmetry excluded for macroscopic Rossby numbers larger than one [20].

Another surprising result is the different behaviour of the different triple correlations. Even though the velocity derivative skewness and the vorticity skewness look similar as statistical descriptors, their evolution in presence of solid-body rotation is far from similar. The former is always damped while the latter shows transient (linear) growth. Furthermore, the initial Gaussianity and isotropy play an important role, especially if linear terms are dominant. For instance, the velocity derivative skewness is zero only if Gaussianity holds, whereas the 
vorticity skewness is zero either because of isotropy or because of Gaussianity.

Finally, the energy decay in the asymptotic long-time limit has been addressed for a selection of runs. It is found to be well described by a power-law $t^{-n}$ regardless of the rotation rate if any. The value of the exponent lies close to $n=10 / 7$ in the isotropic case, whereas it appears to be very close to $n=5 / 7$ in the rotating cases $\mathrm{C} 1$ and $\mathrm{C} 2$ (see Fig. 2). The reduction of $n$ by a factor two in presence of rotation is in perfect agreement with the dimensional analysis by Squires et al. [16].

The numerical results presented here have all been obtained with DNS, a tool that provides easy access to third-order statistics. A drawback of DNS however is of course its limitation to relatively low Reynolds numbers, especially in the case of rotation. Recently, Yang and Domaradzki [24] have studied decaying rotating turbulence at high Reynolds numbers using a Truncated Navier-Stokes (TNS) method. Effectively, TNS consists of a sequence of underresolved DNS runs on a fine mesh with periodic processing of the solution in the range of the modeled subgrid scales. In the asymptotic long-time limit and in absence of rotation, Yang and Domaradzki report an energy decay proportional to power law $t^{-1.2}$, i.e. $n=1.2$, a value that is only slightly smaller than ours $(n \approx 10 / 7)$. It would be interesting to compare the energy decay in presence of rotation ( $n \approx 5 / 7$ in our DNS), but Yang and Domaradzki have not reported energy decay exponents for any of their rotating cases. For the future, it would also be interesting to compare the behaviour of the third-order correlations as obtained here with DNS to that obtained with TNS. Such statistics have not yet been reported for TNS, but the work by Yang and Domaradzki suggests that rotation effects are more pronounced at high Reynolds numbers so one may expect to find a stronger vorticity asymmetry at high Reynolds numbers, consistent with our low Reynolds number results.

Finally, it is remarked that the subtle interplay between linear and nonlinear processes 
is altered in the presence of boundaries, Ekman pumping, or initially coherent structures. Interesting insights to these effects can be found in recent studies by Zavala Sansón and Van Heijst [22], Morize et al. [5], and Davidson and coworkers [28].

\section{Acknowledgments}

This research has been supported by a Marie Curie Fellowship of the European Community programme Marie Curie Training Site on Environmental Turbulence under contract number HPMT-CT-2001-00369. One of the authors (L.J.A.v.B.) would like to thank the Dutch Foundation for Fundamental Research on Matter (FOM) for financial support. We are grateful to C. Morize, P. Staplehurst, and their coworkers for sharing their experimental results. Computational time for the simulations was generously granted by IDRIS, the computing centre of CNRS in France.

\section{Appendix A: RDT and vorticity triple correlations}

The linearised inviscid Navier-Stokes equations in the co-rotating frame follow directly from (1), viz.

$$
\partial_{t} u_{i}=\partial_{i} p-2 \Omega \varepsilon_{i 3 j} u_{j}
$$

Additional useful information on the modified pressure $p$ and on the fluctuating vorticity $\omega_{i}$ is found by taking the divergence and the curl of (A1), respectively, resulting in the Poisson equation

$$
\nabla^{2} p=2 \Omega \omega_{3}
$$


and the linearised inviscid vorticity equation

$$
\partial_{t} \omega_{i}=2 \Omega \partial_{3} u_{i}
$$

The main difficulty in solving equation (A1) comes from the presence of the pressure term which is governed by the Poisson equation (A2) in order to ensure the solenoidal property $\left(\partial_{i} u_{i}=0\right)$. Because of the nonlocal relation between pressure and vorticity,

$$
\nabla^{2} p=g \quad \rightarrow \quad p(\mathbf{x}, t)=-\frac{1}{4 \pi} \int_{\mathbb{R}^{3}} \frac{g\left(\mathbf{x}^{\prime}, t\right)}{\left|\mathbf{x}-\mathbf{x}^{\prime}\right|} \mathrm{d}^{3} \mathbf{x}^{\prime}
$$

the solution of (A1) involves a nonlocal Green's function $\mathcal{G}$, i.e.

$$
u_{i}(\mathbf{x}, t)=\int_{\mathbb{R}^{3}} \mathcal{G}_{i j}\left(\mathbf{x}-\mathbf{x}^{\prime}, t\right) u_{j}\left(\mathbf{x}^{\prime}, 0\right) \mathrm{d}^{3} \mathbf{x}^{\prime}
$$

Similarly, the solution of (A3) is given by (A5) with $u$ replaced by $\omega$, and with a different Green's function. Accordingly, in order to express the solution for the velocity (vorticity) field at position $\mathbf{x}$ and at time $t$, one must know the initial value (here $t=0$ without loss of generality) of the velocity (vorticity) at any position $\mathrm{x}^{\prime}$.

Even though the pressure term is removed from consideration in the vorticity equation (A3), a similar issue comes from the term $\partial_{3} u_{i}$ since it is nonlocal in terms of vorticity (e.g. $\nabla^{2} u_{i}=-\varepsilon_{i j k} \partial_{j} \omega_{k}$ can be compared with $\nabla^{2} p=g$ ). As a consequence, single-point third-order correlations of vorticity (or velocity) at position $\mathrm{x}$ and at time $t$ involve the initial single-point third-order vorticity (or velocity) correlations at three different points, $\mathbf{x}_{1}, \mathbf{x}_{2}$ and $\mathbf{x}_{3}$, say, so that for instance

$$
\begin{aligned}
\left\langle\omega_{i} \omega_{j} \omega_{k}\right\rangle(t)=\int_{\mathbb{R}^{9}} \mathcal{G}_{i p}\left(\mathbf{x}-\mathbf{x}_{1}, t\right) \mathcal{G}_{j q}(\mathbf{x} & \left.-\mathbf{x}_{2}, t\right) \mathcal{G}_{k r}\left(\mathbf{x}-\mathbf{x}_{3}, t\right) \\
& \times\left\langle\omega_{p}\left(\mathbf{x}_{1}, 0\right) \omega_{q}\left(\mathbf{x}_{2}, 0\right) \omega_{r}\left(\mathbf{x}_{3}, 0\right)\right\rangle \mathrm{d}^{3} \mathbf{x}_{1} \mathrm{~d}^{3} \mathbf{x}_{2} \mathrm{~d}^{3} \mathbf{x}_{3} .
\end{aligned}
$$


The problem of nonlocality cannot be avoided using Fourier space. However, the structure of basic linear solutions is simplified in Fourier space since integro-differential equations become algebraic equations. Therefore, consider the Fourier counterparts $\hat{u}_{i}, \hat{p}, \hat{\omega}_{i}$ of the basic variables $u_{i}, p, \omega_{i}$, as depending on the wave number $\mathbf{k}$ which is related to $\mathbf{x}$. The counterpart of the linear solution for $\omega_{i}(\mathbf{x}, t)$ is

$$
\hat{\omega}_{i}(\mathbf{k}, t)=G_{i j}\left(\mathbf{k} / k, \sigma_{k} t\right) \hat{\omega}_{j}(\mathbf{k}, 0)
$$

with

$$
G_{i j}=\Re\left(N_{i} N_{j}^{*} \mathrm{e}^{\imath \sigma_{k} t}\right)
$$

where $\imath^{2}=-1$, see [2]. [Similarly, the counterpart of (A5) is given by (A7) with $\hat{\omega}$ replaced by $\hat{u}$. In Fourier space, however, the Green's function (A8) is valid for both $\hat{u}$ and $\hat{\omega}$.] The definition of the helical mode $N_{\boldsymbol{i}}$, which is normal to $\mathbf{k}$ and depends only on its orientation $\mathbf{k} / k$, is not recalled from Ref. 2 for the sake of brevity. It is only important to recall the unsigned dispersion law of inertial waves, viz. $\sigma_{k}=2 \Omega k_{3} / k$. Of course, the operator $G_{i j}$ is highly anisotropic, changing isotropic statistical symmetry into axial symmetry without mirror symmetry if the initial velocity field is isotropic; but its impact depends on the statistical quantity under consideration. For instance, RDT gives no evolution of second-order velocity (or vorticity) correlations at the same time when the initial velocity field is isotropic. Anyway, the operator $G_{i j}$ is a deterministic operator just like its counterpart in physical space $\mathcal{G}_{i j}$, and it is possible to generate any statistical RDT solution by products of equations (A7). Classically, RDT is used to predict the evolution of second-order statistics, but it is possible to predict correlations of any order, and we will address triple (vorticity) correlations in what follows.

One can readily pass from velocity to vorticity when working in Fourier space since $\boldsymbol{\omega}=$ 
$\imath \mathbf{k} \times \mathbf{u}$. In addition, $\hat{u}_{i}$ and $\hat{\omega}_{i}$ are governed by the same equation (A7) in the case of pure rotation. Thus, triple vorticity correlations in Fourier space display a third-order spectral tensor $\Phi$ as

$$
\left\langle\hat{\omega}_{i}(\mathbf{q}, t) \hat{\omega}_{j}(\mathbf{k}, t) \hat{\omega}_{m}(\mathbf{p}, t)\right\rangle=\Phi_{i j m}(\mathbf{k}, \mathbf{p}, t) \delta(\mathbf{k}+\mathbf{p}+\mathbf{q})
$$

and (A7) yields

$$
\Phi_{i j m}(\mathbf{k}, \mathbf{p}, t)=G_{i r}(-\mathbf{k}-\mathbf{p}, t) G_{j s}(\mathbf{k}, t) G_{m n}(\mathbf{p}, t) \Phi_{r s n}(\mathbf{k}, \mathbf{p}, 0)
$$

Reverting to a single-point correlation, or $\left\langle\omega_{i} \omega_{j} \omega_{m}\right\rangle$, we recover the integral formulation

$$
\left\langle\omega_{i} \omega_{j} \omega_{m}\right\rangle(t)=\int_{\mathbb{R}^{6}} \Phi_{i j m}(\mathbf{k}, \mathbf{p}, t) \mathrm{d}^{3} \mathbf{k} \mathrm{d}^{3} \mathbf{p}
$$

so that for instance

$$
\left\langle\omega_{3}^{3}\right\rangle(t)=\int_{\mathbb{R}^{6}} G_{3 r}(-\mathbf{k}-\mathbf{p}, t) G_{3 s}(\mathbf{k}, t) G_{3 n}(\mathbf{p}, t) \Phi_{r s n}(\mathbf{k}, \mathbf{p}, 0) \mathrm{d}^{3} \mathbf{k} \mathrm{d}^{3} \mathbf{p}
$$

Of course, one must know the contribution of the initial triple correlations for all triads in order to solve (A12), but the problem is now much better documented than it is in physical space. Robust spectral theories such as EDQNM provide a systematic way to express the initially isotropic third-order spectral tensor $\Phi_{i j m}$ in terms of the initial energy spectrum $E(k)$. More generally, advanced EDQNM versions (such as EDQNM2 and EDQNM3 [3, 25]) can be used to solve the fully nonlinear problem in order to replace the initial data $\Phi_{r s n}(\mathbf{k}, \mathbf{p}, 0)$ in $(\mathrm{A} 12)$ by their nonlinear, slowly varying in time, counterpart, but this is another story. A common point of all formulations (linear as well as nonlinear) is the product of three Green's functions (A8) which amounts to phase terms

$$
\mathrm{e}^{22 \Omega t\left( \pm \frac{k_{3}}{k} \pm \frac{p_{3}}{p} \pm \frac{q_{3}}{q}\right)}
$$


as the only time-dependent and rotation-dependent terms in (A12), displaying the triadic resonance condition for interacting inertial waves: $k_{3} / k \pm p_{3} / p \pm q_{3} / q=0$. 


\section{References}

[1] Greenspan, H. P., 1968, The theory of rotating fluids. Cambridge University Press.

[2] Cambon, C. and Jacquin, L., 1989, Spectral approach to non-isotropic turbulence subjected to rotation. J. Fluid Mech., 202, 295-317.

[3] Cambon, C., Mansour, N. N. and Godeferd, F. S., 1997, Energy transfer in rotating turbulence. J. Fluid Mech., 337, 303-332.

[4] Bartello, P., Métais, O. and Lesieur, M., 1994, Coherent structures in rotating three-dimensional turbulence. J. Fluid Mech., 273, 1-29.

[5] Morize, C., Moisy, F. and Rabaud, M., 2005, Decaying grid-generated turbulence in a rotating tank. Phys. Fluids, 17, 095105.

[6] Cambon, C. and Scott, J. F., 1999, Linear and nonlinear models of anistropic turbulence. Annu. Rev. Fluid Mech., 31, 1-53.

[7] Gence, J.-N. and Frick, C., 2001, Birth of the triple correlations of vorticity in an homogeneous turbulence submitted to a solid body rotation. C. R. Acad. Sci. Paris Série IIB, 329, 351-356.

[8] Rogallo, R. S., 1981, Numerical experiments in homogeneous turbulence. NASA Tech. Mem., 81315

[9] Liechtenstein, L., Godeferd, F. S. and Cambon, C., 2005, Nonlinear formation of structures in rotating stratified turbulence. $J o T, \mathbf{6}, 1-21$.

[10] Vincent, A. and Meneguzzi, M., 1991, The spatial structure and statistical properties of homogeneous turbulence. $J$. Fluid Mech., 225, 1-20.

[11] Batchelor, G. K. and Townsend, A. A., 1947, Decay of vorticity in isotropic turbulence. Proc. Roy. Soc. A, 191, 534-550.

[12] McComb, W. D., 1990, The Physics of Fluid Turbulence, Oxford Engineering Science Series 25. Clarendon Press, Oxford.

[13] Morize, C., Moisy, F., Rabaud, M. and Sommeria, J., 2006, On the cyclone-anticyclone asymmetry in decaying rotating turbulence. Proceedings of the Conference on Turbulence and Interactions 
TI2006, Porquerolles, France, May 29 - June 2.

[14] Saffman, P. G., 1967, The large-scale structure of homogeneous turbulence. J. Fluid Mech., 27, $581-593$.

[15] Skrbek, L. and Stalp, S. R., 2000, On the decay of homogeneous isotropic turbulence. Phys. Fluids, 12, 1997-2019.

[16] Squires, K. D., Chasnov, J. R., Mansour, N. N. and Cambon, C., 1994, The asymptotic state of rotating homogeneous turbulence at high Reynolds numbers. Proceedings of the 74th Fluid Dynamics Symposium on Application of Direct and Large Eddy Simulation to Transition and Turbulence, Chania, Greece, AGARD Conf. Proc. 551, 4-1.

[17] Morize, C., Moisy, F., 2006, Energy decay of rotating turbulence with confinement effects. Phys. Fluids, 18, 065107.

[18] Zhou, Y., 1995, A phenomenological treatment of rotating turbulence. Phys. Fluids, 7, $2092-2094$.

[19] Godeferd, F. S. and Lollini, L., 1999, Direct numerical simulations of turbulence with confinement and rotation. J. Fluid Mech., 393, 257-308.

[20] Jacquin, L., Leuchter, O., Cambon, C. and Mathieu, J., 1990, Experimental study of homogeneous turbulence in the presence of rotation. J. Fluid Mech., 220, 1-52.

[21] Staplehurst, P. J., Davidson, P. A. and Dalziel, S.B., 2007, Structure formation in homogeneous freely-decaying rotating turbulence. Under consideration for publication in J. Fluid Mech.

[22] Zavala Sansón, L. and Van Heijst, G. J. F., 2000, Nonlinear Ekman effects in rotating barotropic flows. J. Fluid Mech., 412, 75-91.

[23] Bourouiba, L. and Bartello, P., 2007, The intermediate Rossby number range and 2D-3D transfers in rotating decaying homogeneous turbulence. Under consideration for publication in J. Fluid. Mech.

[24] Yang, X. and Domaradzki, J. A., 2004, Large-eddy simulations of decaying rotating turbulence. Phys. Fluids, 16, 4088.

[25] Cambon, C., Rubinstein, R. and Godeferd, F. S., 2004, Advances in wave turbulence: rapidly 
rotating flows. New Journal of Physics, 6, 1-29.

[26] Galtier, S., 2003, Weak inertial-wave turbulence theory. Phys. Rev. E, 68, 015301-1-4.

[27] Van Bokhoven, L. J. A., 2007, Experiments on rapidly rotating turbulent flows. PhD thesis. Eindhoven University of Technology, Eindhoven.

[28] Davidson, P. A., Staplehurst, P. J. and Dalziel, S. B., 2006, On the evolution of eddies in a rapidly rotating system. J. Fluid Mech., 557, 135-144. 
Table 1. Overview of the performed runs. For each run are given the number of computation points in physical space $N$, the initial Reynolds number $\operatorname{Re}(0) \equiv \nu^{-1}$ (with $\nu$ the nondimensional kinematic viscosity), the duration of the precalculation $t_{\mathrm{ini}}$, the Coriolis parameter $f \equiv 2 \Omega$, the initial Taylor-based Reynolds number $\operatorname{Re}_{\lambda}\left(t_{\text {ini }}\right) \equiv u^{\prime} \lambda / \nu$, and the initial Taylor-based Rossby number $\operatorname{Ro}_{\lambda}\left(t_{\text {ini }}\right) \equiv u^{\prime} /(2 \Omega \lambda)$, with $u^{\prime} \equiv\left\langle u_{i}^{2} / 3\right\rangle^{1 / 2}$ the characteristic velocity and $\lambda \equiv\left(15 u^{\prime 2} /\left\langle\omega_{i}^{2}\right\rangle\right)^{1 / 2}$ the Taylor length scale. Note that our values for $R e_{\lambda}\left(t_{\mathrm{ini}}\right)$ are somewhat smaller than the typical values of 60 to 80 often used in DNS of forced homogeneous turbulence with $128^{3}$ resolution.

\begin{tabular}{lcccccc}
\hline & & & & & & \\
Run & $N$ & $R e(0)$ & $t_{\text {ini }}$ & $f$ & $R e_{\lambda}\left(t_{\text {ini }}\right)$ & $R o_{\lambda}\left(t_{\text {ini }}\right)$ \\
& & & & & & \\
\hline A & 144 & 600 & 5.0 & $5.0 \pi$ & 26.7 & 0.073 \\
B1 & 288 & 1200 & 2.0 & $5.0 \pi$ & 39.2 & 0.36 \\
B2 & 288 & 1200 & 4.0 & $5.0 \pi$ & 33.2 & 0.14 \\
B3 & 288 & 1200 & 6.0 & $5.0 \pi$ & 30.3 & 0.084 \\
B4 & 288 & 1200 & 8.0 & $5.0 \pi$ & 28.6 & 0.057 \\
C1 & 288 & 1200 & 4.0 & $0.5 \pi$ & 33.2 & 1.41 \\
C2 & 288 & 1200 & 4.0 & $2.5 \pi$ & 33.2 & 0.28 \\
C3 & 288 & 1200 & 4.0 & $5.0 \pi$ & 33.2 & 0.14 \\
C4 & 288 & 1200 & 4.0 & $10 \pi$ & 33.2 & 0.071 \\
D & 540 & 3000 & 2.0 & $5.0 \pi$ & 46.6 & 0.37 \\
\hline & & & & & & \\
\hline
\end{tabular}




\section{Figure captions}

Fig. 1: Time evolution of the kinetic energy $E$ for various viscosities; case A: $\nu=1 / 600$, case C3: $\nu=1 / 1200$, case D: $\nu=1 / 3000$.

Fig. 2: Kinetic energy $E$ as a function of time $t$. The thick solid line represents the isotropic precalculation for $t<4$, and the reference computation for $t \geq 4$ (Cref). The thin solid line (C1) and dash-dotted (C2) line both represent decaying rotating turbulence, with the background rotation is abruptly imposed at $t=4$. In the asymptotic limit of large $t$, the energy falls off as $E \propto t^{-10 / 7}$ in the isotropic case and as $E \propto t^{-5 / 7}$ in presence of rotation.

Fig. 3: (a,b) Time evolution of the Taylor-based Reynolds number $R e_{\lambda}$ and the Taylor-based Rossby number $R o_{\lambda}$, respectively, for various viscosities; case $\mathrm{A}: \nu=1 / 600$, case C3: $\nu=$ $1 / 1200$, case D: $\nu=1 / 3000$.

Fig. 4: $(\mathrm{a}, \mathrm{b}, \mathrm{c})$ Energy spectrum $E(k)$ of the decaying rotating turbulence at $t=t_{f}$ for various viscosities; case A: $\nu=1 / 600$, case C3: $\nu=1 / 1200$, case D: $\nu=1 / 3000$. Energy spectra of the decaying isotropic turbulence at times $t=t_{\text {ini }}$ and $t_{f}$ are also shown.

Fig. 5: (a) Time evolution of the normalised vortex stretching term $e^{*}$ during and after the isotropic precalculation for various viscosities; case A: $\nu=1 / 600$, case C3: $\nu=1 / 1200$, case D: $\nu=1 / 3000$. Background rotation in cases A, C3, and D is applied at time $t_{\mathrm{ini}}=5.0,4.0$, and 2.0, respectively. For the sake of reference, the isotropic precalculations have been prolonged. (b) Log-log plot showing the vorticity skewness $S_{\omega_{3}}$ as a function of the scaled and shifted time $\tau$ for cases $\mathrm{A}, \mathrm{C} 3$ and $\mathrm{D}$.

Fig. 6: As Fig. 5, but now for various durations of the isotropic precalculation; case B1: $t_{\text {ini }}=2.0$, case B2: $t_{\text {ini }}=4.0$, case B3: $t_{\text {ini }}=6.0$, case B $4: t_{\text {ini }}=8.0$. 
Fig. 7: As Fig. 5, but now for various background rotation rates; case $\mathrm{C} 1: f \equiv 2 \Omega=0.5 \pi$, case $\mathrm{C} 2: f=2.5 \pi$, case $\mathrm{C} 3: f=5 \pi$, case $\mathrm{C} 4: f=10 \pi$. In all cases background rotation is applied at time $t_{\text {ini }}=4.0$.

Fig. 8: Time evolution of the minimal set of single-point vorticity triple correlations in rotating turbulence. Various background rotation rates are shown; $\mathrm{C} 1: f \equiv 2 \Omega=0.5 \pi, \mathrm{C} 2: f=2.5 \pi$, C3: $f=5 \pi, \mathrm{C} 4: f=10 \pi$. All triple correlations are normalised by $\left\langle\omega_{3}^{2}\right\rangle^{3 / 2}$.

Fig. 9: (a) Probability distribution functions $p\left(\omega_{i}\right)$ as a function of the vorticity component $\omega_{i}$ at times $t=4$ (solid lines) and $t=8$ (thin lines). (b) As panel (a) but now the normalised pdf $p\left(\omega_{i}\right) \sigma\left(\omega_{i}\right)$ is displayed as a function of the normalised vorticity component $\omega_{i} / \sigma\left(\omega_{i}\right)$, with $\sigma\left(\omega_{i}\right)$ the standard deviation of $p\left(\omega_{i}\right)$. All data corresponds to purely decaying isotropic homogeneous turbulence.

Fig. 10: Normalised probability distribution functions $p\left(\omega_{i}\right) \sigma\left(\omega_{i}\right)$ as a function of the normalised vorticity component $\omega_{i} / \sigma\left(\omega_{i}\right)$ for times $t=t_{\mathrm{ini}}=4$ (solid lines) and $t=t_{r}$ (thin lines), with $\sigma\left(\omega_{i}\right)$ the standard deviation of $p\left(\omega_{i}\right)$. Panels (a) and (b) correspond to cases C1 and $\mathrm{C} 2$, respectively.

Fig. 11: Normalised probability distribution functions of vorticity $p\left(\omega_{i}\right) \sigma\left(\omega_{i}\right)$ as a function of normalised vorticity component $\omega_{i} / \sigma\left(\omega_{i}\right)$ for times $t=t_{\mathrm{ini}}=4$ (solid lines) and $t=t_{r}$ (thin lines), with $\sigma\left(\omega_{i}\right)$ the standard deviation of $p\left(\omega_{i}\right)$. From top to bottom, $i=1,2,3$. The corresponding numerical time, scaled time, and Taylor-scale based Reynolds and Rossby number, are as follows (for as far as relevant). Case $\mathrm{C} 1: t=17.12, \tau=6.56, R e_{\lambda} \approx 44.8$, $R o_{\lambda} \approx 0.10 ;$ case $\mathrm{C} 2: t=6.94, \tau=7.36, R e_{\lambda} \approx 45.5, R o_{\lambda} \approx 0.11$; case C3: $t=5.95, \tau=4.88$, $R e_{\lambda} \approx 45.5, R o_{\lambda} \approx 0.07 ;$ case $\mathrm{C} 4: t=5.18, \tau=5.91, R e_{\lambda} \approx 43.1, R o_{\lambda} \approx 0.04$; isotropic case Cref: $t=7.98, R e_{\lambda} \approx 33.2$. 
Fig. 12: Time evolution of the componential gradient skewness $S_{\partial_{1} u_{1}}, S_{\partial_{2} u_{2}}$ and $S_{\partial_{3} u_{3}}$ for various viscosities (cases $\mathrm{A}, \mathrm{C} 3$ and $\mathrm{D}$ ), various durations of the isotropic precalculation (cases B1-B4), and various background rotation rates (cases $\mathrm{C} 1-\mathrm{C} 4$ ). The minus sign in front of $S_{\partial_{\alpha} u_{\alpha}}$ is introduced to enable direct comparison with the vortex stretching term $e^{*}$ shown in Figs 5-7(a). Relevant parameter values of the various cases are summarised in Table 1.

Fig. 13: Vorticity skewness $S_{\omega_{3}}$ as a function of the number of tank rotations $\Omega t / 2 \pi$ reported by Morize et al. [5] for decaying rotating grid-generated turbulence. Five data sets are shown: $\star-f \equiv 2 \Omega=0.26, R e_{g}=4.7 \times 10^{4}, R o_{g}=120 ; \diamond-f=1.06, R e_{g}=3.1 \times 10^{4}, R o_{g}=19 ;$ $\circ-f=3.0, R e_{g}=3.1 \times 10^{4}, R o_{g}=6.8 ; \bullet-f=3.0, R e_{g}=6.2 \times 10^{4}, R o_{g}=14 ; \square-$ $f=8.6, R e_{g}=3.1 \times 10^{4}, R o_{g}=2.4$. Here, the grid-based Reynolds number $R e_{g} \equiv V_{g} M / \nu$, and the grid-based Rossby number $R o_{g} \equiv V_{g} /(f M)$, with $V_{g}$ the maximum grid velocity and $M$ the mesh size. The dashed line is proportional to power law $(\Omega t)^{0.6}$. With gratitude to Morize and coworkers.

Fig. 14: Vorticity skewness $S_{\omega_{3}}$ as a function of the normalised time $t / \Omega$ as reported by Staplehurst et al. [21] for decaying rotating grid-generated turbulence $\left[t_{\text {ini }}=0\right.$ in their laboratory experiments $(\mathrm{LE})]$. Four data sets are shown: $-f \equiv 2 \Omega=1.88, R e_{g}=83, R o_{g}=2.7$; - $f=1.88, R e_{g}=130, R o_{g}=2.0 ; \diamond-f=3.88, \quad R e_{g}=83, \quad R o_{g}=1.4 ; \circ-$ $f=3.88, R e_{g}=130, R o_{g}=1.0$. Here, the grid-based Reynolds number $R e_{g} \equiv u^{\prime} b / \nu$, and the grid-based Rossby number $R o_{g} \equiv\left\langle\omega_{x}^{2}\right\rangle^{1 / 2} / \Omega$, with $u^{\prime} \equiv\left\langle u_{i}^{2} / 3\right\rangle^{1 / 2}$ the root-meansquare velocity, $b=M / 5$ the bar size, $M$ the mesh size, and $\omega_{x}$ the integral scale vorticity for one of the directions perpendicular to $\Omega / \Omega$. The experimental results by Staplehurst et al. may be compared to our DNS case $\mathrm{C} 1-f=0.5 \pi, R e \equiv u^{\prime} \mathcal{L} / \nu=102, R o \equiv u^{\prime} /(2 \Omega \mathcal{L})=0.46$, with $\mathcal{L} \equiv E^{-1} \int E(k) / k \mathrm{~d} k$ the integral length scale. The experimental and numerical results 
are qualitatively the same, except for a shift in time. With gratitude to Staplehurst and coworkers. 


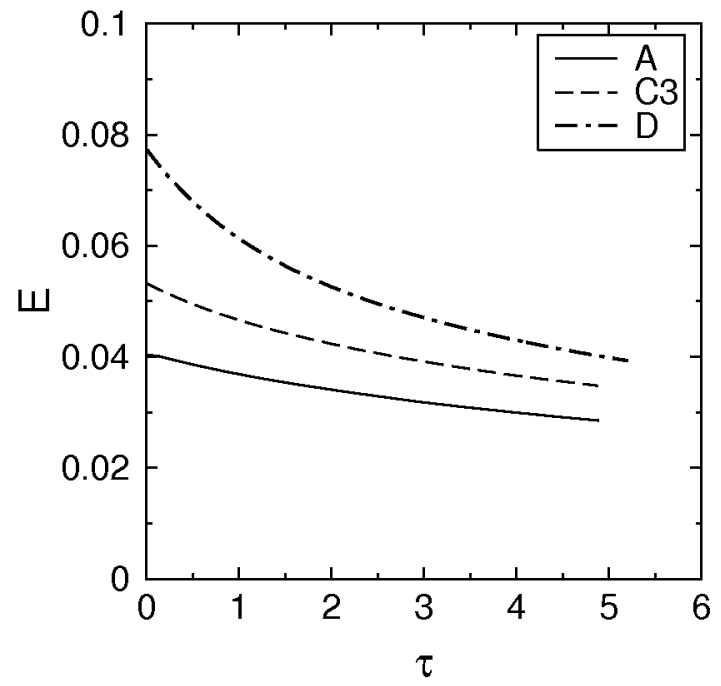

Figure 1. 


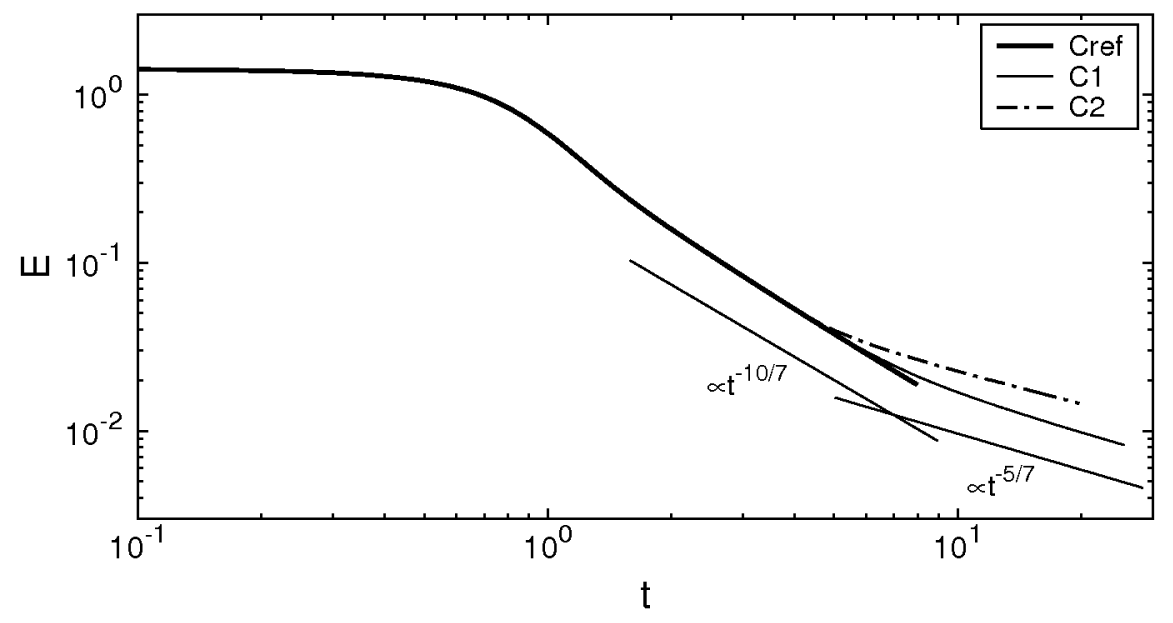

Figure 2 . 

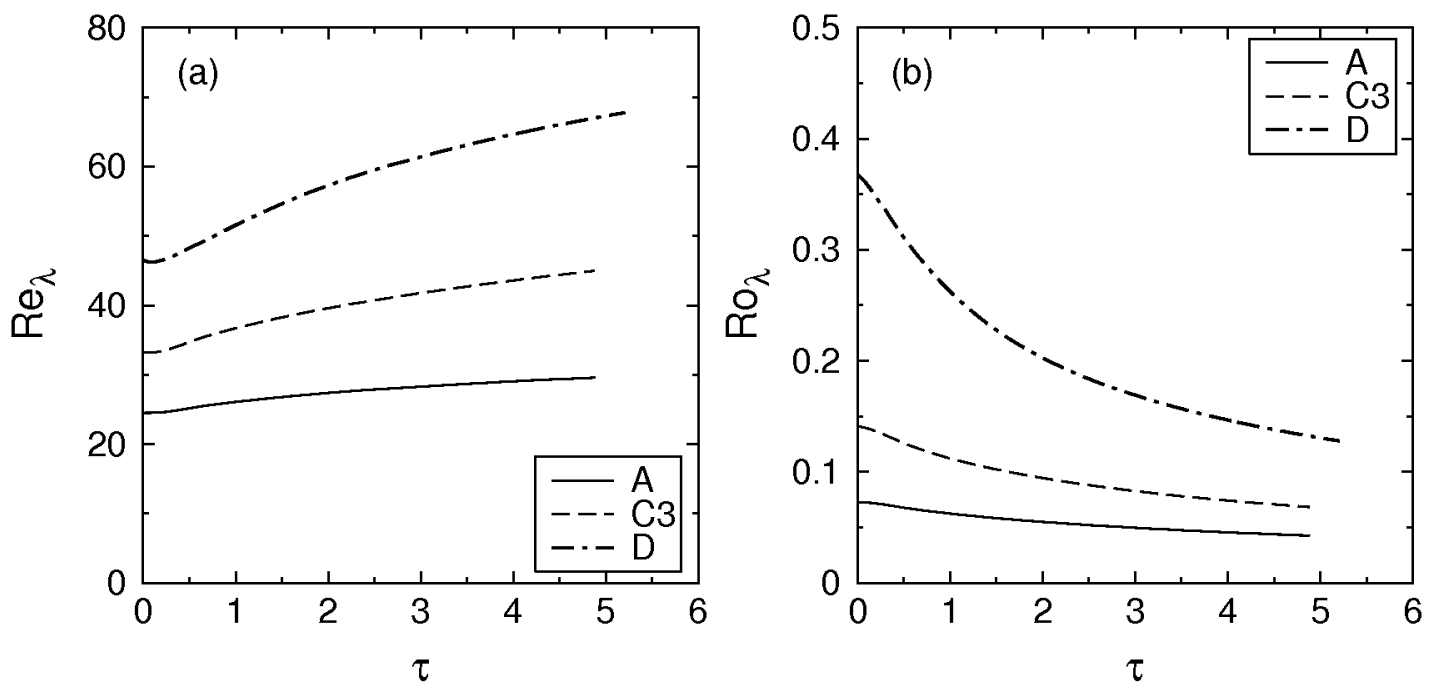

Figure 3. 

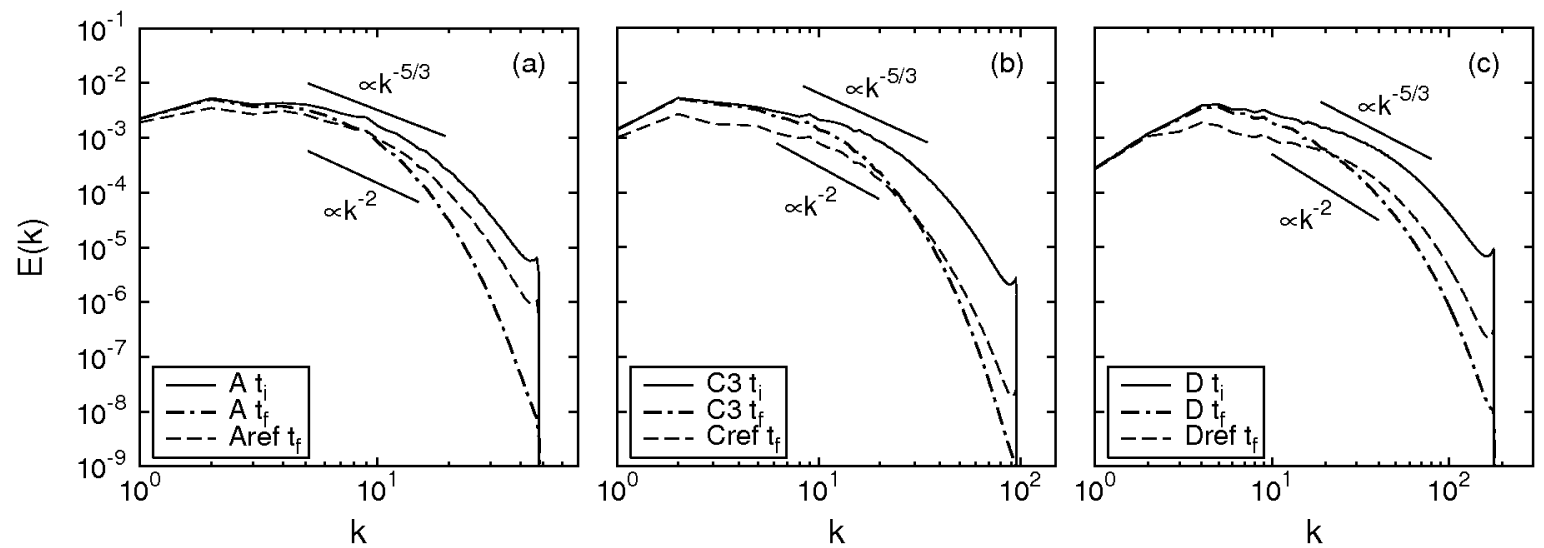

Figure 4. 

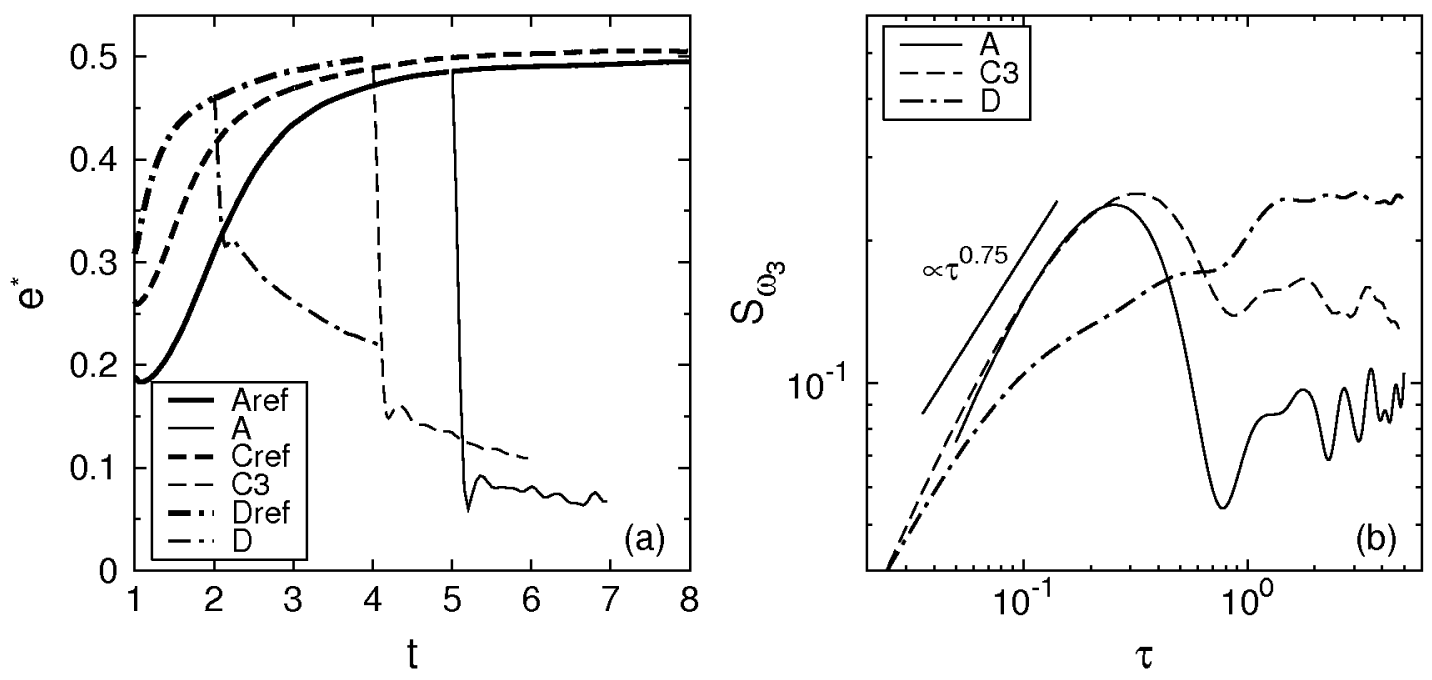

Figure 5 . 

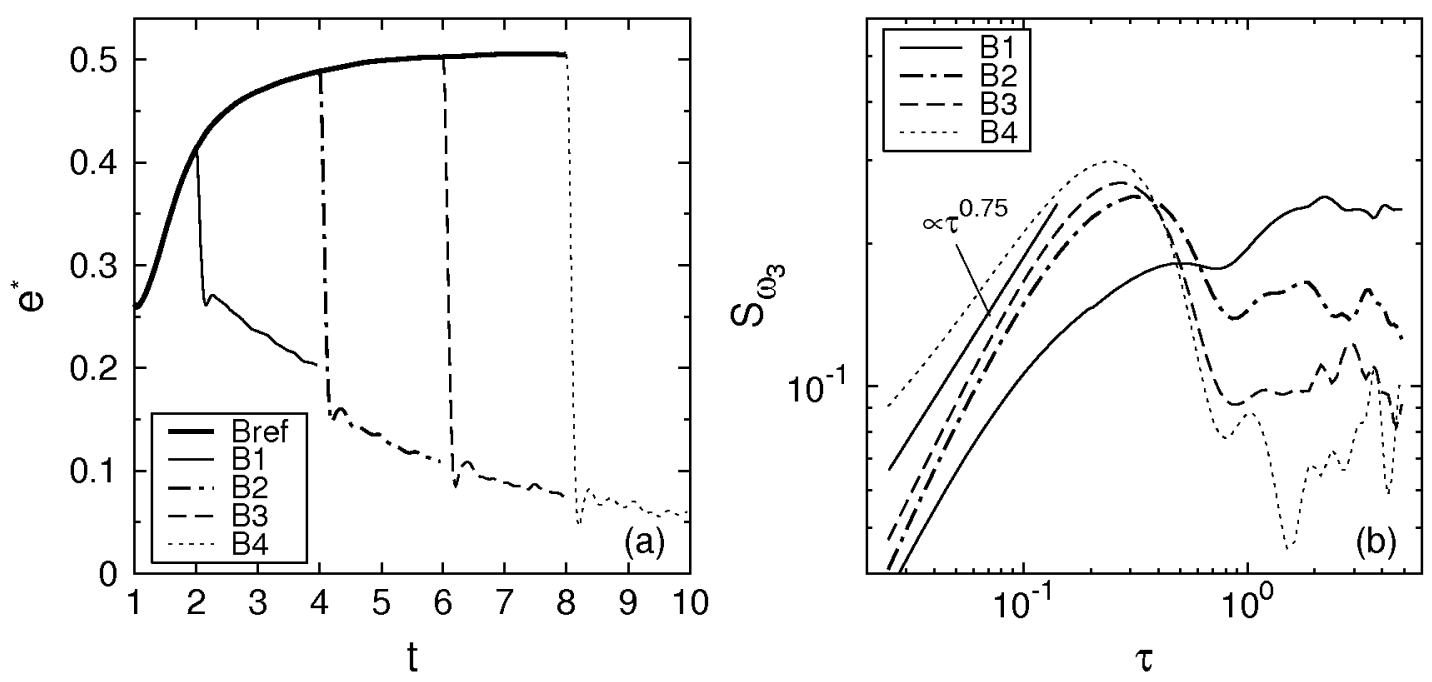

Figure 6. 

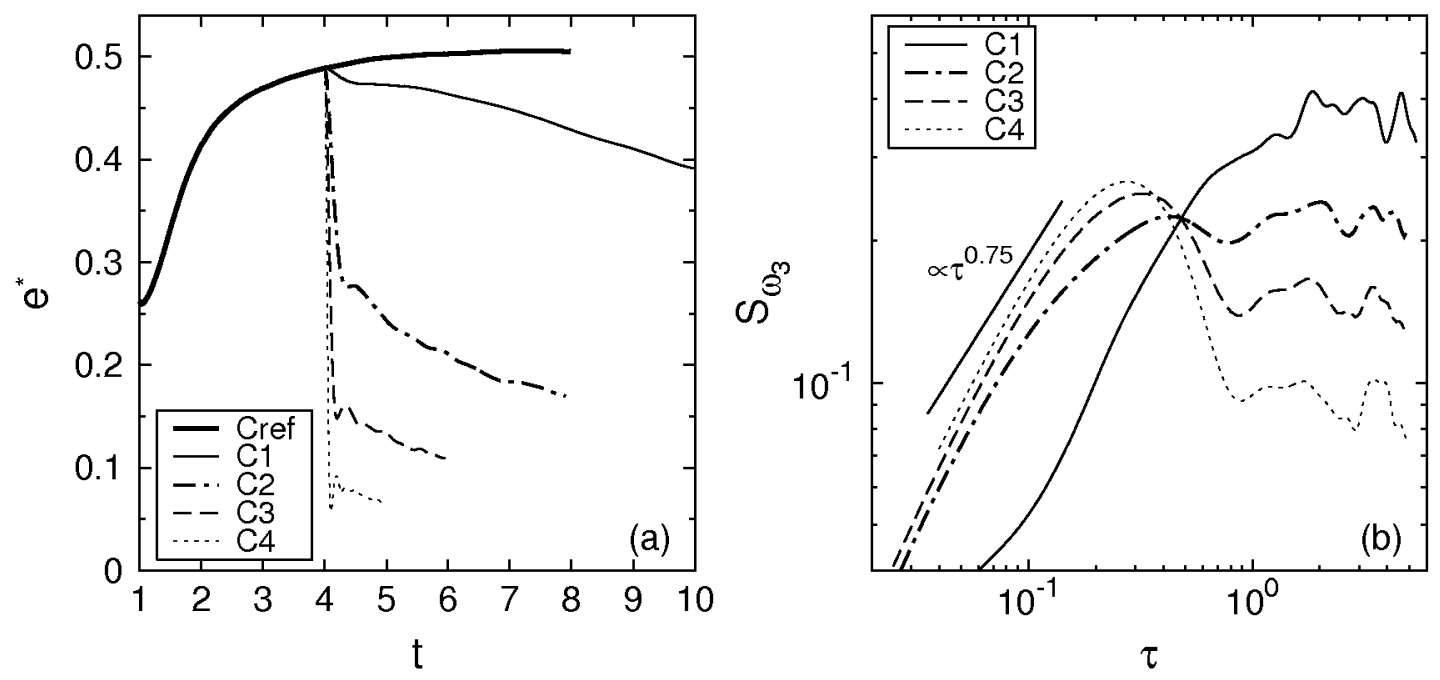

Figure 7. 

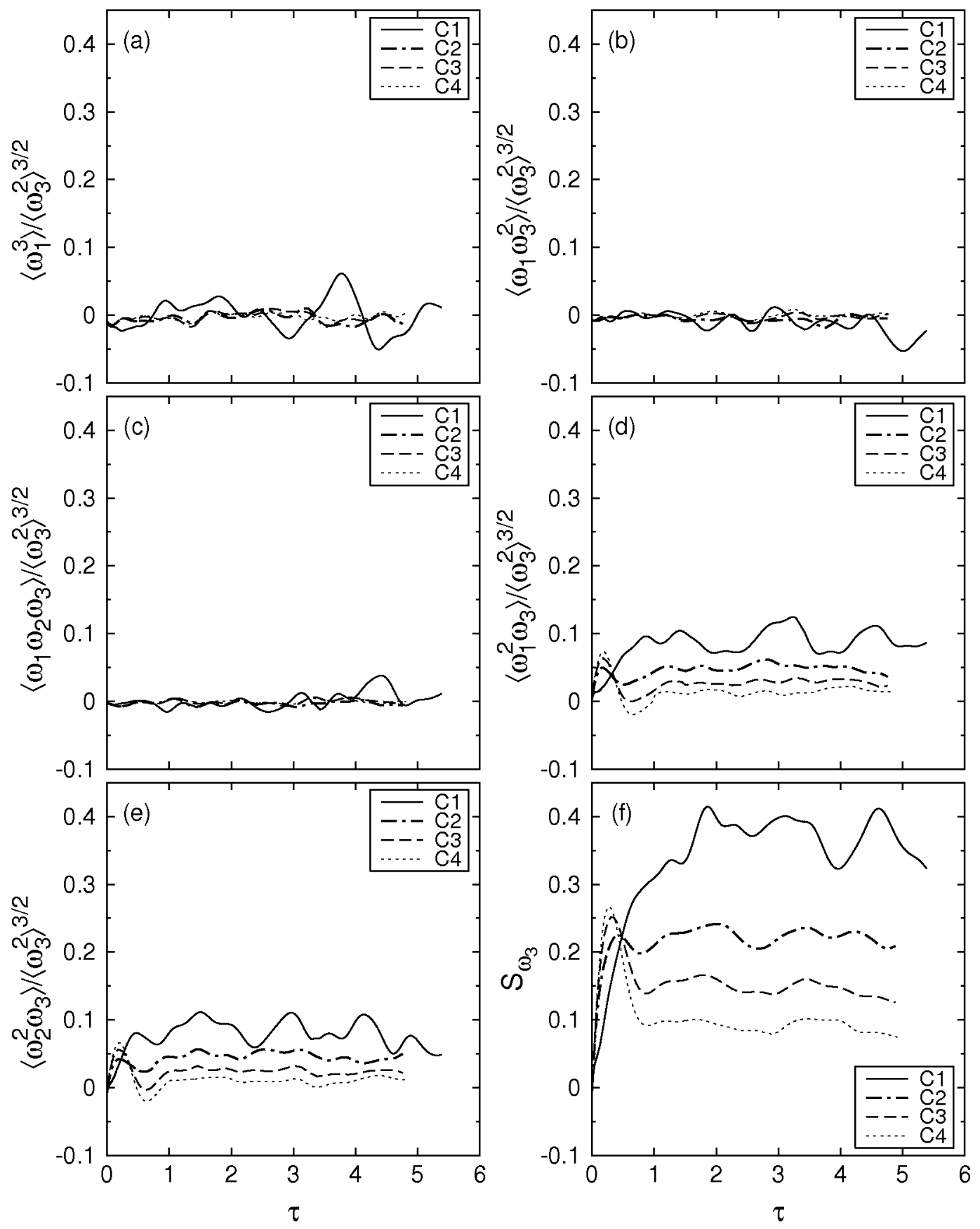

Figure 8. 

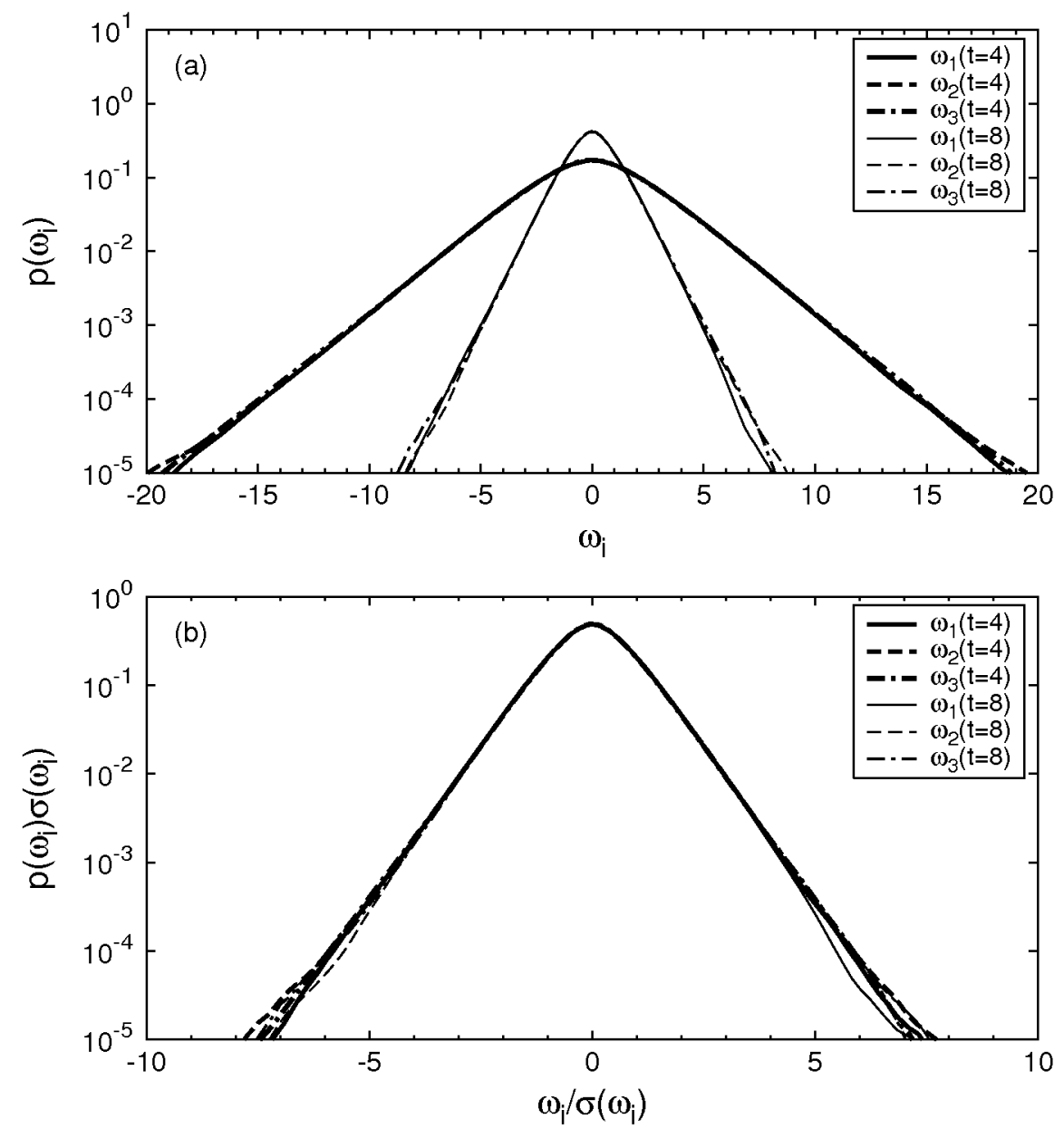

Figure 9. 

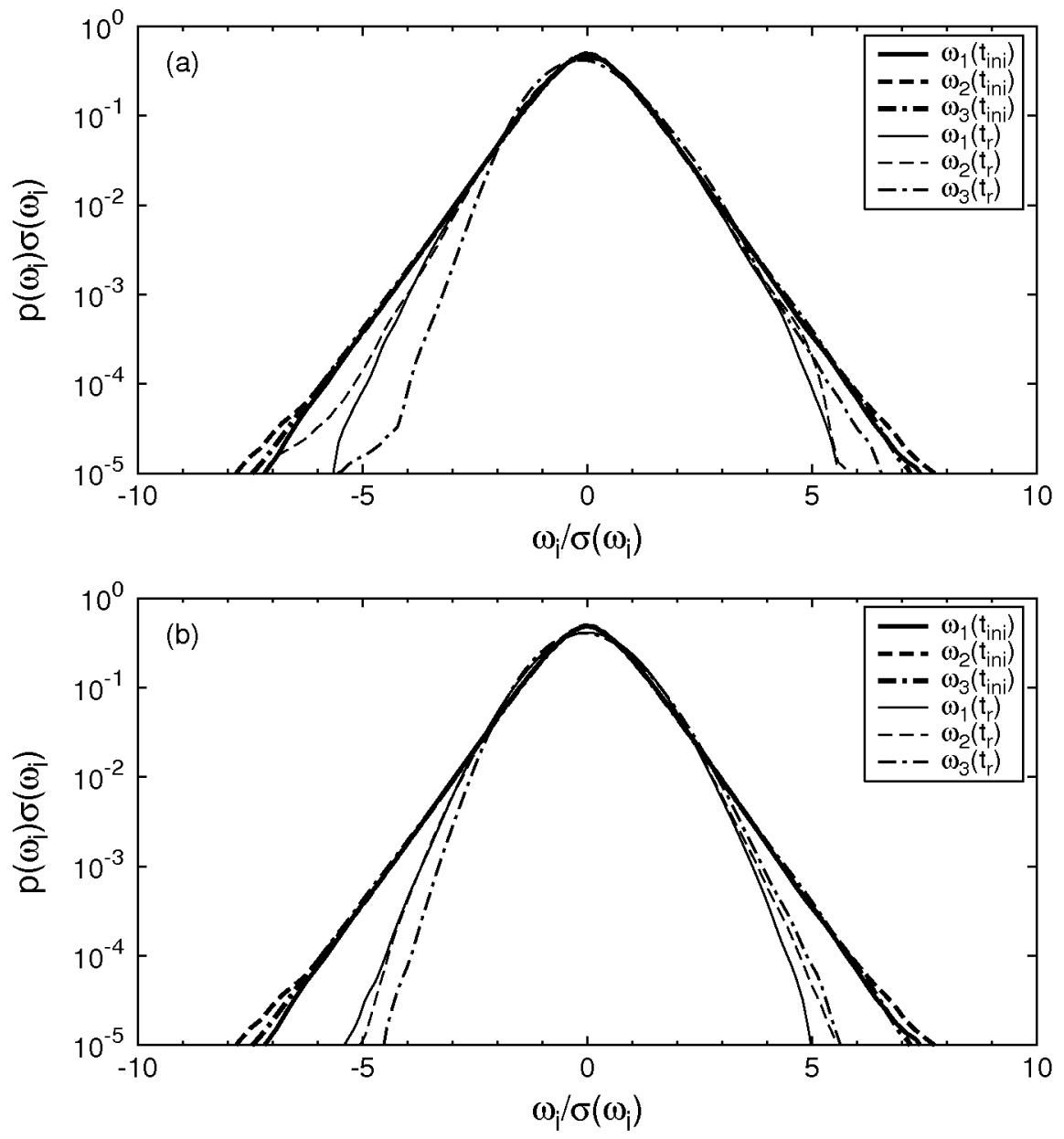

Figure 10. 

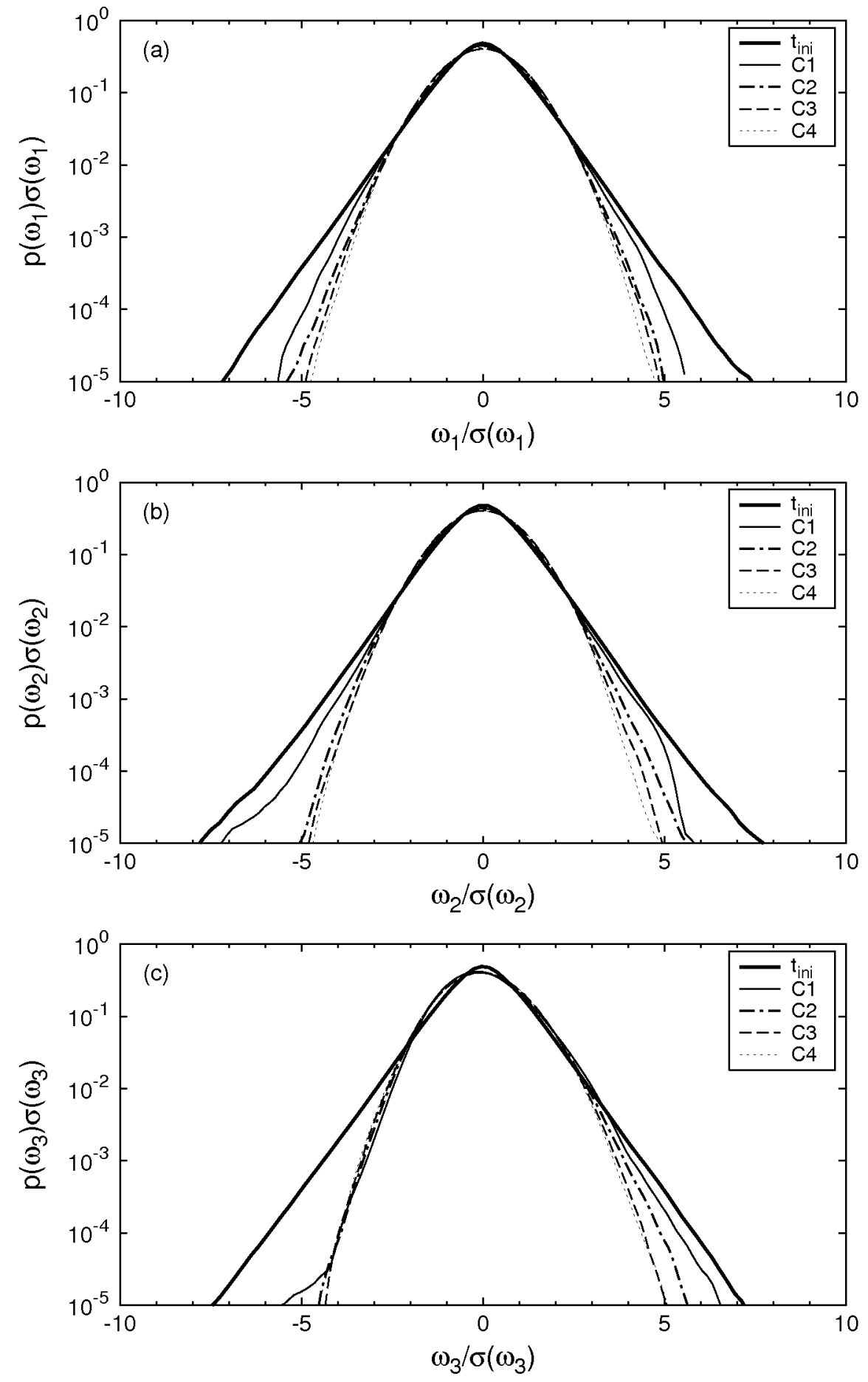

Figure 11. 

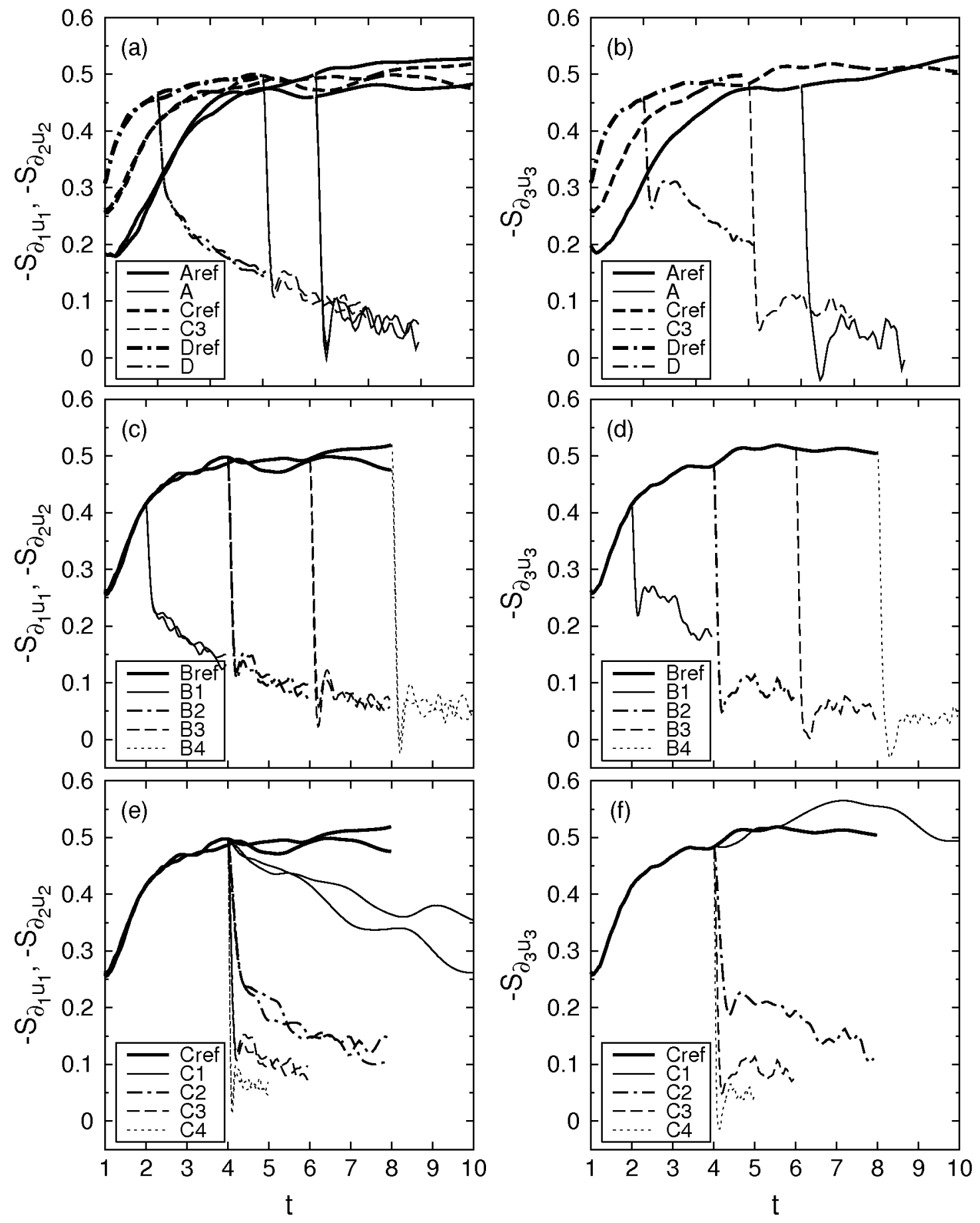

Figure 12. 


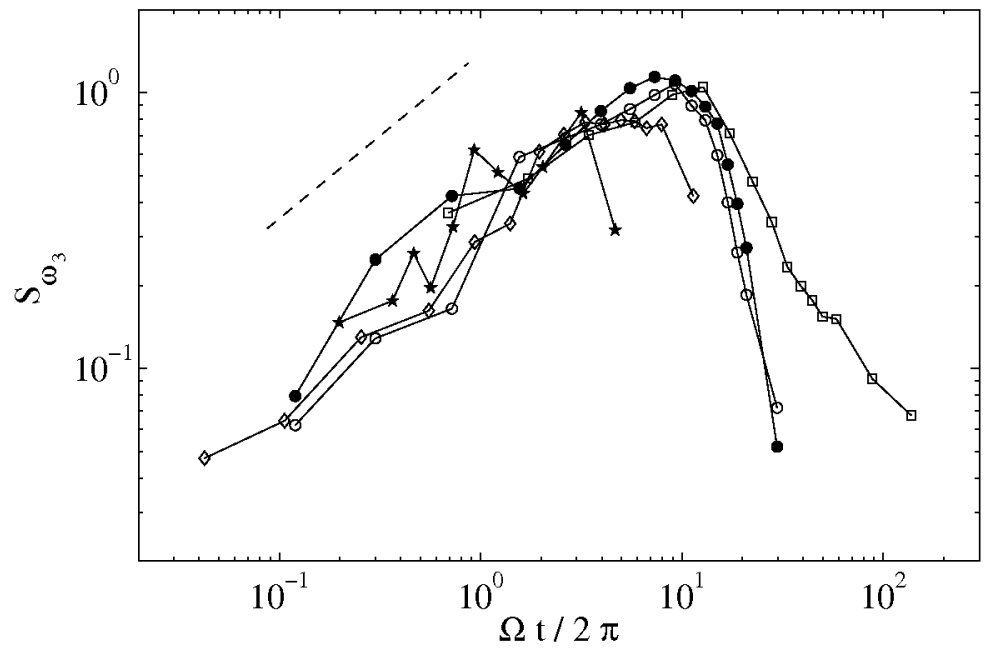

Figure 13. 


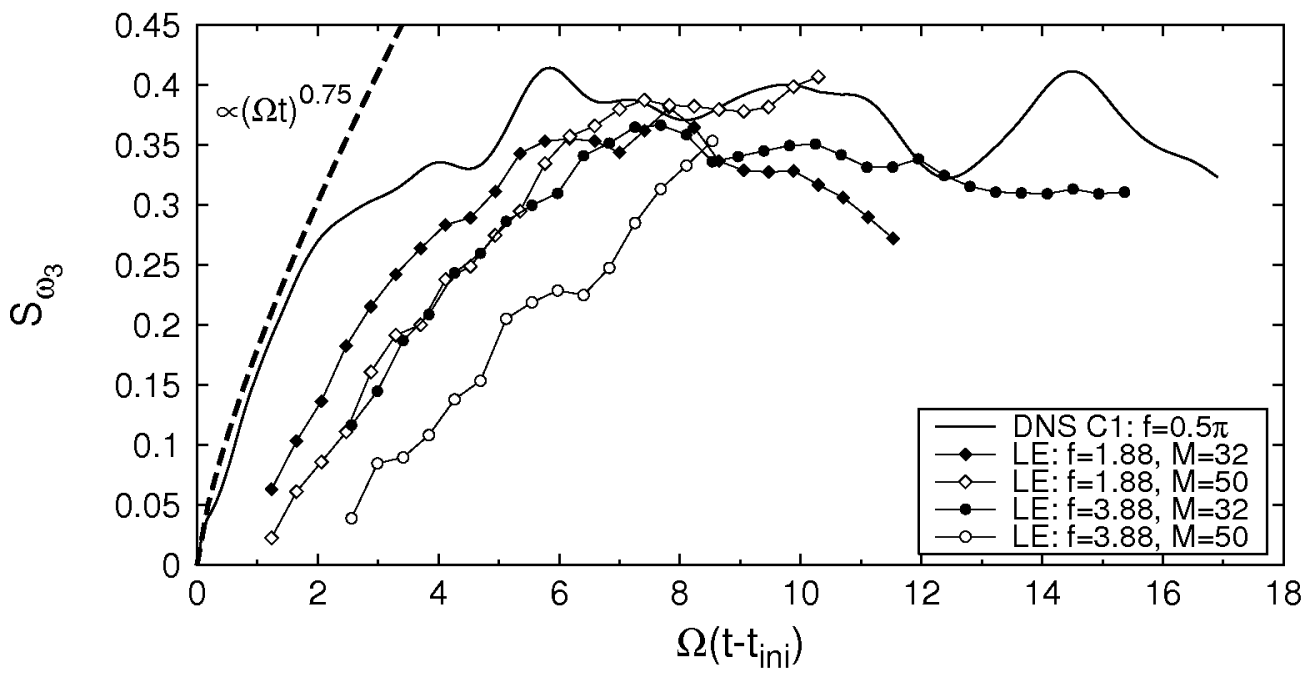

Figure 14. 